\title{
Posttranslational arginylation enzyme Ate1 affects DNA mutagenesis by regulating stress response
}

\author{
Akhilesh Kumar ${ }^{1}$, Michael D Birnbaum¹, Devang M Patel ${ }^{1}$, William M Morgan ${ }^{1}$, Jayanti Singh², Antoni Barrientos ${ }^{3,4}$ and \\ Fangliang Zhang ${ }^{*, 1,5}$
}

Arginyltransferase 1 (Ate1) mediates protein arginylation, a poorly understood protein posttranslational modification (PTM) in eukaryotic cells. Previous evidence suggest a potential involvement of arginylation in stress response and this PTM was traditionally considered anti-apoptotic based on the studies of individual substrates. However, here we found that arginylation promotes cell death and/or growth arrest, depending on the nature and intensity of the stressing factor. Specifically, in yeast, mouse and human cells, deletion or downregulation of the ATE1 gene disrupts typical stress responses by bypassing growth arrest and suppressing cell death events in the presence of disease-related stressing factors, including oxidative, heat, and osmotic stresses, as well as the exposure to heavy metals or radiation. Conversely, in wild-type cells responding to stress, there is an increase of cellular Ate1 protein level and arginylation activity. Furthermore, the increase of Ate1 protein directly promotes cell death in a manner dependent on its arginylation activity. Finally, we found Ate1 to be required to suppress mutation frequency in yeast and mammalian cells during DNA-damaging conditions such as ultraviolet irradiation. Our study clarifies the role of Ate1/ arginylation in stress response and provides a new mechanism to explain the link between Ate1 and a variety of diseases including cancer. This is also the first example that the modulation of the global level of a PTM is capable of affecting DNA mutagenesis. Cell Death and Disease (2016) 7, e2378; doi:10.1038/cddis.2016.284; published online 29 September 2016

As a common response to changing environmental cues, posttranslational modifications (PTMs) of proteins can promptly modulate cellular functions without de novo translation or transcription. Among them, arginylation is the posttranslational addition of an extra arginine to an existing peptide chain, usually to the $\mathrm{N}$ terminus, thus capable of changing the surface charge as well as the primary sequence of the target. ${ }^{1}$ Arginylation is mediated by arginyltransferase 1 (Ate1), ${ }^{2,3}$ an evolutionarily conserved enzyme found in eukaryotic organisms and some bacteria. ${ }^{4-6}$ Nearly a hundred proteins have been found to be arginylated and the list of identified substrates is continuously growing, ${ }^{7,8}$ suggesting a widespread effect of this PTM in vivo.

Mounting evidence has suggested an involvement of arginylation in stress response, a naturally occurring process undertaken by cells in stressing conditions, which often lead to growth arrest or cell death. For more than two decades, researchers have repeatedly observed signs of increased arginylation activity in tissues of animals (such as rat, chicken and fish) following a variety of insults including nerve crush injury or whole-body hyperthermia. ${ }^{9-18}$ At the molecular level, arginylation is often observed on proteins that are nitrosylated, oxidized or misfolded, which are common consequences of stress. ${ }^{19-21}$ However, the exact nature of arginylation's involvement in these processes remains unclear. According to the $\mathrm{N}$-end rule, a protein degradation theory that correlates the metabolic stabilities of a peptide to the identity of its $\mathrm{N}$-terminal residue, arginylation constitutes an acute degradation signal conferring a half-life of $<3 \mathrm{~min}^{22}$ For this reason, previous studies mainly focused on identifying novel substrates for cues of mechanistic insights of arginylation, under the assumption that this modification should lead to a quick degradation. On this basis, several studies suggested that a normal activity of arginylation is required for anti-apoptotic activities through proteins such as drosophila inhibitor of apoptosis protein, and degradation of pro-apoptotic fragments of RIPK1 (receptorinteracting serine/threonine protein kinase 1) and BRCA1 (breast cancer 1, early onset) created by the action of caspase or calpain. ${ }^{23-27}$ Therefore, Ate1-mediated arginylation has been traditionally considered to have an anti-apoptotic effect in vivo. ${ }^{23,27-29}$ However, arginylation affects a broad range of proteins with diverse functions, some of which are arguably anti-apoptotic. ${ }^{30}$ Further complicating the matter, arginylation may regulate protein structure and function independently of protein half-life. ${ }^{31-35}$ Hence, it may be ineffective or inaccurate to ascribe a global function to Ate1 based on the identities of a few individual substrates. A new approach is needed

\footnotetext{
${ }^{1}$ Department of Molecular and Cellular Pharmacology, University of Miami Leonard M. Miller School of Medicine, Miami, FL 33136, USA; ${ }^{2}$ Department of Medicine, Division of Cardiology, University of Miami Leonard M. Miller School of Medicine, Miami, FL 33136, USA; ${ }^{3}$ Department of Neurology, University of Miami Leonard M. Miller School of Medicine, Miami, FL 33136, USA; ${ }^{4}$ Department of Biochemistry and Molecular Biology, University of Miami Leonard M. Miller School of Medicine, Miami, FL 33136, USA and ${ }^{5}$ Sylvester Comprehensive Cancer Center, University of Miami Leonard M. Miller School of Medicine, Miami, FL 33136, USA

${ }^{*}$ Corresponding author: F Zhang, Department of Molecular and Cellular Pharmacology or Sylvester Comprehensive Cancer Center, University of Miami Leonard M. Miller School of Medicine, 1600 NW 10th Avenue, RMSB 6047, Miami, FL 33136, USA. Tel: +305 2430 159; Fax: +305 2434 555; E-mail: fzhang2@miami.edu Abbreviations: Ate1, arginyltransferase 1; CFU, colony-forming cells per OD unit; EdU, 5-ethynyl-2'-deoxyuridine; GFP, green fluorescent protein; HFF, human foreskin fibroblast; KO, knockout; MEF, mouse embryonic fibroblast; mAte1, mammalian Ate1; OD, optical density; RT, room temperature; shRNA, short hairpin RNA; STS, staurosporine; TUNEL, Terminal deoxynucleotidyl transferase dUTP nick end labeling; UV, ultraviolet; WT, wild type

Received 05.2.16; revised 01.8.16; accepted 10.8.16; Edited by G Ciliberto
} 
to directly evaluate the overall molecular impact of Ate1mediated arginylation regardless of individual substrates.

To test the role of Ate1 and arginylation in stress response, we utilized multiple eukaryotic systems including Saccharomyces cerevisiae (budding yeast), mouse cells and human cells, all of which contain the evolutionarily conserved ATE1 gene. We found that, when arginylation activity is globally downregulated by the deletion or silencing of the ATE1 gene, cellular sensitivities to a variety of stressing factors were significantly reduced, resulting in bypass of growth arrest or reduction of cell death under stress. In addition, the Ate1 protein level and the global arginylation activity are increased in cells under stress, and Ate1 mediates cell death with an arginylation-dependent manner. Finally, Ate1 is needed for suppressing the outcome of DNA mutagenesis during DNAdamaging stress. To our knowledge, our finding is the first example of a PTM having a global effect on DNA mutagenesis.

\section{Results}

Removal or downregulation of Ate1 disrupts stress response and reduces cellular sensitivity to a variety of stressing conditions. Ate 1 is coded by a single gene in yeast and mammals. ${ }^{4,5}$ When we deleted the evolutionarily conserved ATE1 gene in the budding yeast, $S$. cerevisiae (strain BY4741, unless otherwise indicated), we found no obvious effect on growth in non-stressing conditions in nutrient-rich medium (Figures 1a and b). Upon exposure to stress, including $\mathrm{H}_{2} \mathrm{O}_{2}$-induced oxidative stress, heavy metals, high salt or high temperature, wild-type (WT) yeast grew at a significantly lower rate compared with non-stressing conditions, which is an expected outcome of normal stress response (Figures 1a and b). However, the growth of ate $1 \Delta$ yeast was less affected by these stressors (Figures 1a and b), suggesting a disruption of stress response. ${ }^{36}$ The deletion of the ATE1 gene in a different yeast strain, W303, similarly increased cellular resistance to $\mathrm{CdCl}_{2}$ (Figure 1c).

To test whether Ate1 has a similar role in mammals, we compared WT and ATE1-KO mouse embryonic fibroblasts (MEFs) and found that deletion of the ATE1 gene increased cellular resistance to stressors such as cellular oxidant $\mathrm{H}_{2} \mathrm{O}_{2}$, heavy metal $\mathrm{CdCl}_{2}$ and microbial alkaloid toxin staurosporine (STS; Figure 1d). In addition, when ATE1 expression was attenuated by short hairpin RNA (shRNA)-mediated knockdown in MEFs and human foreskin fibroblasts (HFFs), resistance to the oxidant $\mathrm{H}_{2} \mathrm{O}_{2}$ was increased (Figures 1e and $\mathrm{f}$ ).

Common effects of stress response include cell death and growth arrest. To test whether the deletion of ATE1 affects cell death in yeast, we examined cellular viabilities by colonyforming per cells optical density (OD) unit (CFU) in yeast cultures in the presence of lethal doses of $\mathrm{H}_{2} \mathrm{O}_{2}$. We found that ate $1 \Delta$ yeast cultures had higher percentages of viable cells compared with the WT (Figure 2a). Furthermore, using the terminal deoxynucleotidyl transferase dUTP nick end labeling (TUNEL) assay to probe apoptosis, a programmed cell death event, we found that the deletion of ATE1 greatly attenuated $\mathrm{H}_{2} \mathrm{O}_{2}$-induced apoptosis (Figure $2 \mathrm{~b}$ ), which contradicts the prevailing hypothesis for the anti-apoptotic roles for Ate1 and arginylation. $^{23,28,29}$
To examine whether Ate1 also has an impact on growth arrest, we exposed yeast cultures to a moderate concentration $(150 \mu \mathrm{M})$ of $\mathrm{CdCl}_{2}$. This treatment led to a much lower growth rate in the WT yeast culture compared with ate1 1 (Figure 2c). However, both cultures had similar viabilities as assessed by the CFU assay (Figure 2d). In fact, in the late sampling time point $(24 \mathrm{~h})$, the WT yeast even appeared to have slightly higher viability compared with ate $1 \Delta$, probably due to nutrient constraints in the ate $1 \Delta$ yeast culture because they reached a saturation density at that time point. Therefore, the faster growth rates of ate $1 \Delta$ yeast in stressing condition was likely caused by a lack of growth arrest.

To further test whether Ate1 affects both growth arrest and cell death for the same stressor, we challenged the WT and ate $1 \Delta$ yeast with high-temperature conditions. At $40^{\circ} \mathrm{C}$, a heat-stress temperature for yeast, the ate1 $\Delta$ yeast grew significantly faster than the WT. However, when these yeast cultures were transferred from $40^{\circ} \mathrm{C}$ to room temperature (RT), a non-stressing temperature for recovery, similar numbers of colonies eventually formed in both the ate $1 \Delta$ and WT yeast. This suggests that both yeast strains were equally viable, and their difference in growth at $40{ }^{\circ} \mathrm{C}$ was mainly due to a difference in growth arrest (Figure 2e). However, when yeast were incubated at $42^{\circ} \mathrm{C}$ and then transferred to RT, ate $1 \Delta$ yeast were able to form significantly more colonies than the WT yeast, indicating that a deletion of ATE1 in this condition yielded a higher cell survival rate (Figure 2f). Therefore, the lack of Ate1 may lead to the bypass of growth arrest and/or the suppression of cell death from the same stressor, dependent on the intensity of stressor.

To test whether mammalian Ate1 (mAte1) mediates apoptosis, we challenged WT and ATE1-KO MEFs with apoptosisinducing reagent STS of relatively high doses (200-1000 nM) for a short duration ( $5 \mathrm{~h}$ ). By employing the Annexin- $\mathrm{V}$ staining assay to detect early apoptotic signals, we found that the knockout (KO) of ATE1 gene in MEFs significantly reduced apoptotic rates in the presence of STS (Figure 3a). Similar results were observed with a different stressor, $\mathrm{CdCl}_{2}$ (Figure 3a). Next, to test whether mAte1 regulates growth arrest in stressing conditions, we challenged the WT and ATE1-KO MEFs with either $\mathrm{H}_{2} \mathrm{O}_{2}$ or $\mathrm{CdCl}_{2}$, with lower doses and longer durations (12 h). Using a thymidine analog, 5-ethynyl-2'-deoxyuridine (EdU) to measure cell proliferation activity, we found no significant difference between WT and ATE1-KO MEFs in non-stressing conditions. However, in the presence of stressors, the percentage of EdU-positive population in WT cells is significantly lower than in ATE1-KO MEFs, suggesting that the absence of ATE1 prevents growth suppression of mammalian cells under stress (Figure $3 b$ ).

Ate1 and arginylation are upregulated during stress and are responsible for cell death. In yeast and mammals, only one ATE1 gene was identified. ${ }^{4,5}$ To confirm that the deletion of this gene can abolish cellular arginylation activity, we designed a reporter substrate termed DD- $\beta 15-G F P$ (Figure $4 a$ ). When this reporter protein was expressed in yeast cells, we found that its arginylated form existed only in WT yeast, but not in ate $1 \Delta$ (Figure 4b). A similar observation was made in mammalian MEFs (Figure 4e). As a minor note, the steady-state level of the reporter protein, as probed by anti- 
a

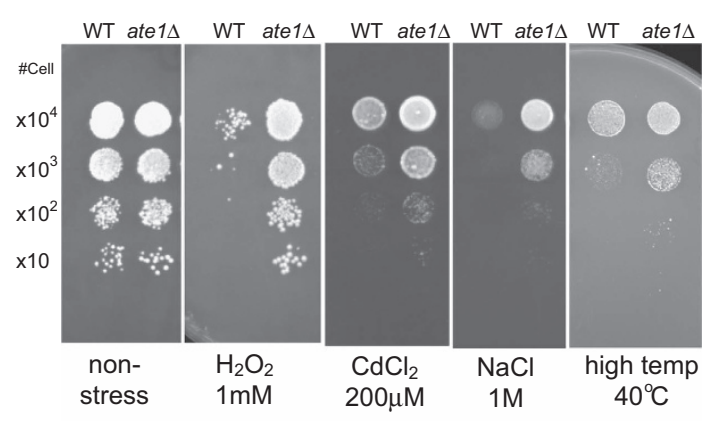

C W303-strain yeast

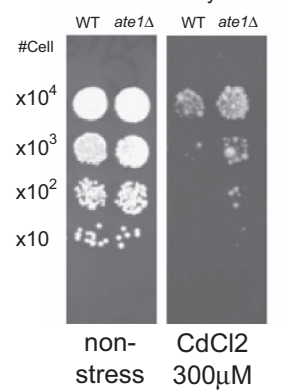

BY4741-strain yeast

d

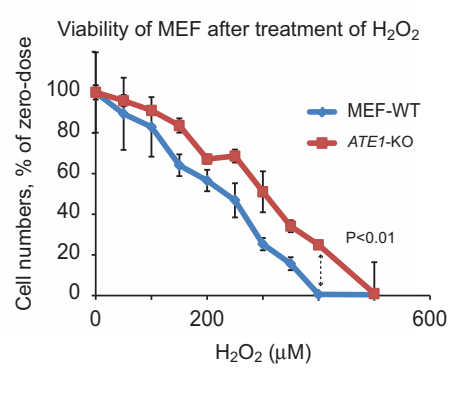

b
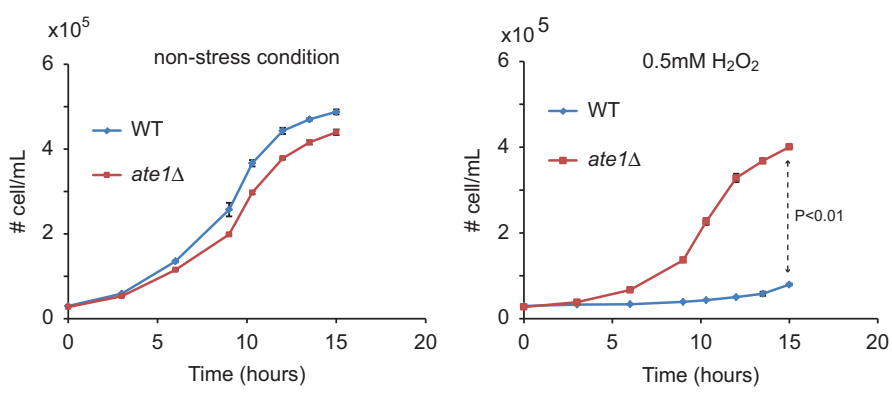

e

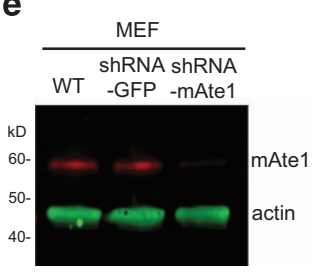

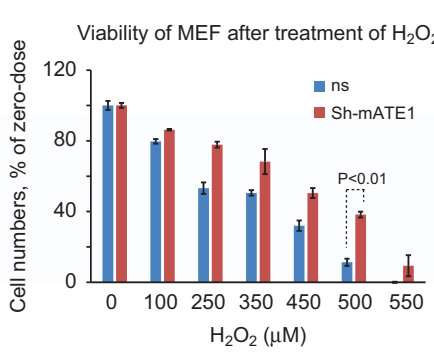
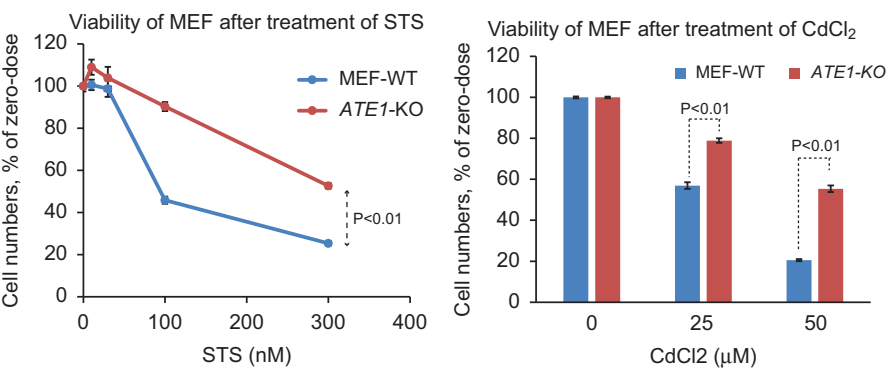
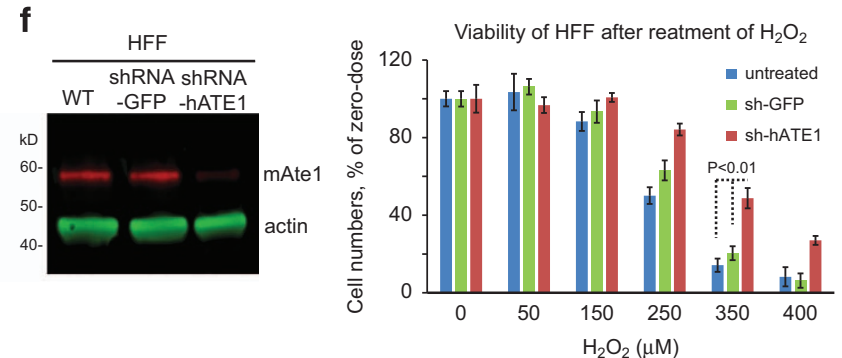

Figure 1 Knockout or knockdown of ATE1 decreases cellular sensitivity towards stressing conditions. (a) Growth test using serial dilutions of wild-type (WT) and ate1 $\Delta$ yeast (S. cerevisae, BY4741 strain, unless otherwise mentioned) in non-stressing condition or in the presence of one of the following stressors: common cellular oxidant $\left(\mathrm{H}_{2} \mathrm{O}_{2}, 1 \mathrm{mM}\right)$, heavy metal $\left(\mathrm{CdCl}_{2}, 200 \mu \mathrm{M}\right)$, high salt $(\mathrm{NaCl}, 1 \mathrm{M})$ or high temperature $\left(40{ }^{\circ} \mathrm{C}\right)$. For $\mathrm{H}_{2} \mathrm{O}_{2}$ treatment, cells were plated on SD plates with glucose. For the other treatments and the non-stressing controls, cells were plated on YPD plates. (b) Growth curves of WT and ate1 $\Delta$ yeast cultured in liquid media, in the presence or absence of the oxidative stressor $\mathrm{H}_{2} \mathrm{O}_{2}$. Error bars represent S.D., $n=3$. (c) Growth test using WT or ate1 $\Delta$ yeast (strain W303) in non-stressed conditions or in the presence of the heavy metal $\mathrm{CdCl}_{2}$ $(300 \mu \mathrm{M})$. (d) Viabilities of WTor ATE1-KO mouse embryonic fibroblasts (MEFs) after $12 \mathrm{~h}$ treatments with increasing concentrations of cellular oxidant $\mathrm{H}_{2} \mathrm{O}_{2}$, bacterial toxin STS or heavy metal $\mathrm{CdCl}_{2}$. The number of viable cells after $\mathrm{H}_{2} \mathrm{O}_{2}$ treatment was measured by Calcein $\mathrm{AM}$, a cellular dye that emits fluorescence only in live cells. The number of viable cells after STS and $\mathrm{CdCl}_{2}$ treatments was directly counted with an automated cell counter (TC-20 from Bio-Rad) with the cross-staining of Trypan blue, which stains dead cell but not live cells. In each type of cell, one sample of cells with control treatment (DMSO only) was used as normalization for other samples, for WTor ATE1-KO, respectively. Error bar represents S.E.M. of multiple repeats ( $n=4$ for $\mathrm{H}_{2} \mathrm{O}_{2}$ and STS, and $n=3$ for $\mathrm{Cdcl}_{2}$ ). (e) Immunoblot analysis of the steady-state levels of mammalian Ate1 (mAte1) in MEF transfected with either shRNA specific against mouse ATE1 (sh-mATE1) or shRNA against GFP (used as a non-targeting control). The band of mAte1 was probed with a monoclonal antibody (Millipore, clone \#6F11) recognizing all four major splicing variants of Ate1 in mammalian cells, which are almost identical in molecular sizes and poorly understood in functional differences. Actin was used as a loading control. The right panel graph shows the quantification of viability of MEFs transfected with Ate1 knockdown or non-targeting control, after the treatment with $\mathrm{H}_{2} \mathrm{O}_{2}$ for $12 \mathrm{~h}$, as measured by the cellular viability indicator Calcein AM. Error bars represent S.E.M. ( $\left.n=4\right)$. (f) Similar to e, except that human foreskin fibroblast (HFF) and shRNA against human ATE1 (sh-hATE1) were used. Error bars represent SEM $(n=4)$. Throughout this study, the $P$-value was calculated by two-tailed Student's $t$-test between specific data points, unless otherwise indicated

GFP, was obviously lower in WT yeast than in ate1 $\Delta$, likely due to an arginylation-mediated degradation in yeast (Figure $4 b$ ).

In most past studies, arginylation was demonstrated by radioactively labeled arginine, which can be incorporated into protein by arginylation or translation. To separate these two effects, translation was inhibited by either inhibitors or depletion of ribosomes. ${ }^{9-14}$ However, most translation inhibitors are unfortunately leaky, and removal of ribosomes can collaterally deplete Ate1 due to their known affinity. ${ }^{37}$ To remove these ambiguities, we used DD- $\beta 15-G F P$ for an 'in-lysate' reaction to examine arginylation activity in cell extracts (Figure 4c, left panel). Using this assay, we found an increase of arginylation signal in WTyeast exposed to $\mathrm{NaCl}$ as an osmotic stressor, in proportion to stress durations (Figure 4c, right panel). Also, the level of the substrate is reduced proportionally, likely due to arginylation-mediated degradation (Figure 4c, right panel and middle strip). As a control, extracts from ate1 $1 \Delta$ yeast exposed to the same 
treatment did not show an increase (or any signal) of the arginylated reporter or a reduction of the substrates (Supplementary Figure S1), suggesting that the stress-induced increase of arginylation depends on the presence of Ate1. Consistent with the observation in yeast, a dose-dependent increase of arginylation activity was found in MEFs treated with $\mathrm{H}_{2} \mathrm{O}_{2}$ (Figure 4e). These data indicate that the global arginylation activity in the cell is upregulated as part of the natural stress response process, which is consistent with previous observations. $^{9-14}$

To test whether Ate1 protein is also upregulated in stress response, we took advantage of a commercially available

\section{a}

Viability\% of WT or ate $1 \Delta$-yeasts

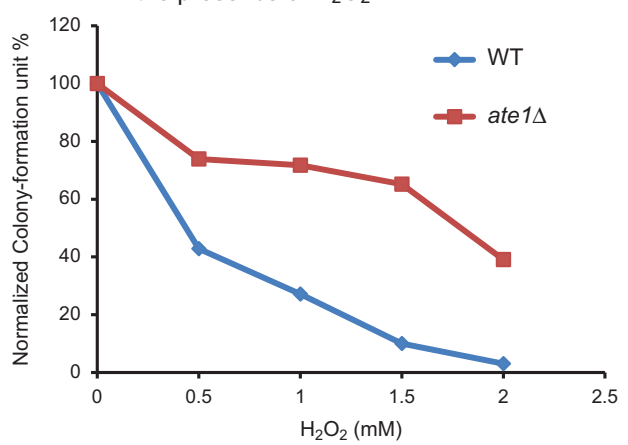

C

Growth curves of WT or ate1 $1 \Delta$-yeasts in either non-stress condition or in the presence of $150 \mu \mathrm{M} \mathrm{CdCl}_{2}$
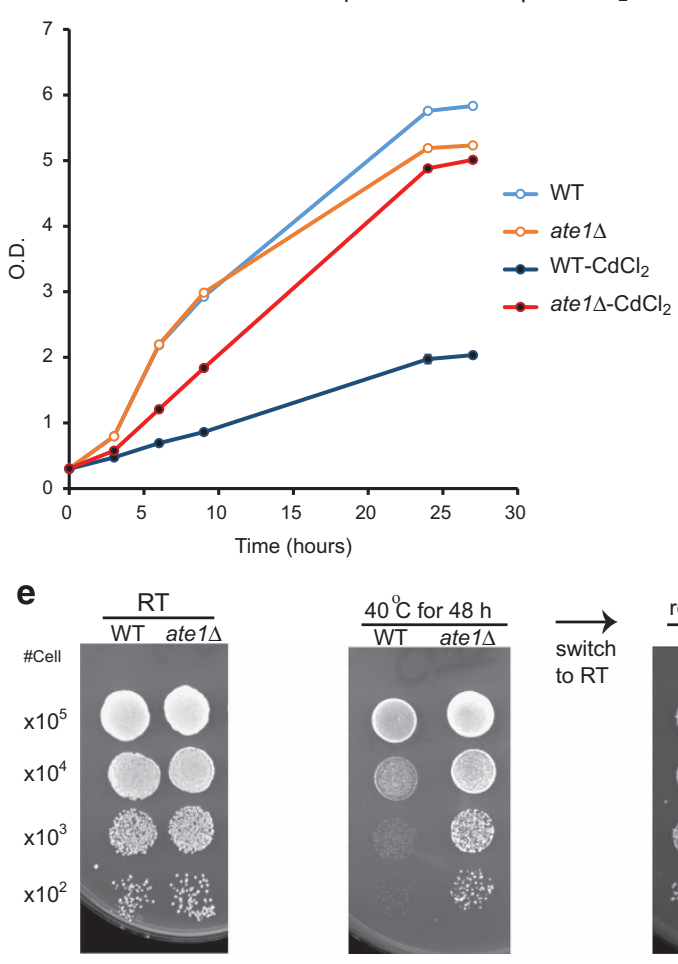

f
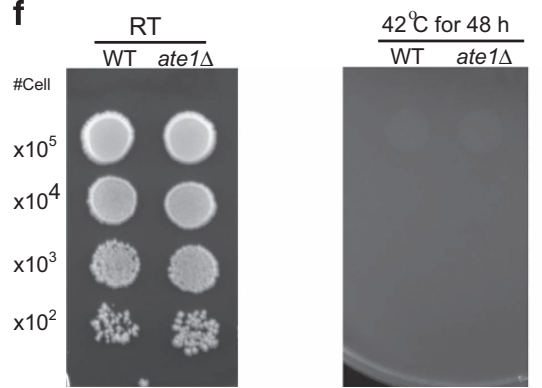

b

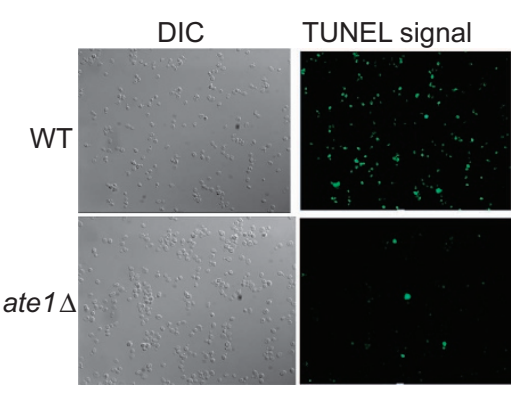

$\mathrm{H}_{2} \mathrm{O}_{2} 0.5 \mathrm{mM}$

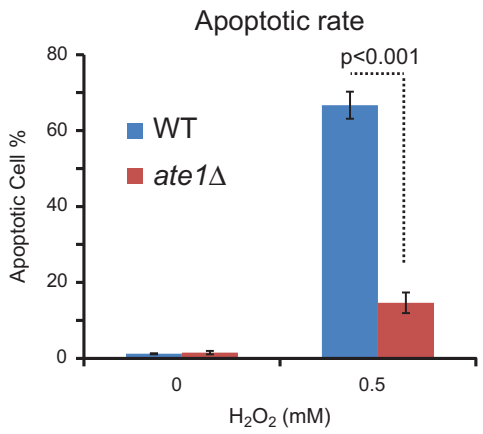

d

d Viability\% of WT or ate $1 \Delta$-yeasts in the presence of $150 \mu \mathrm{M} \mathrm{CdCl} 2$
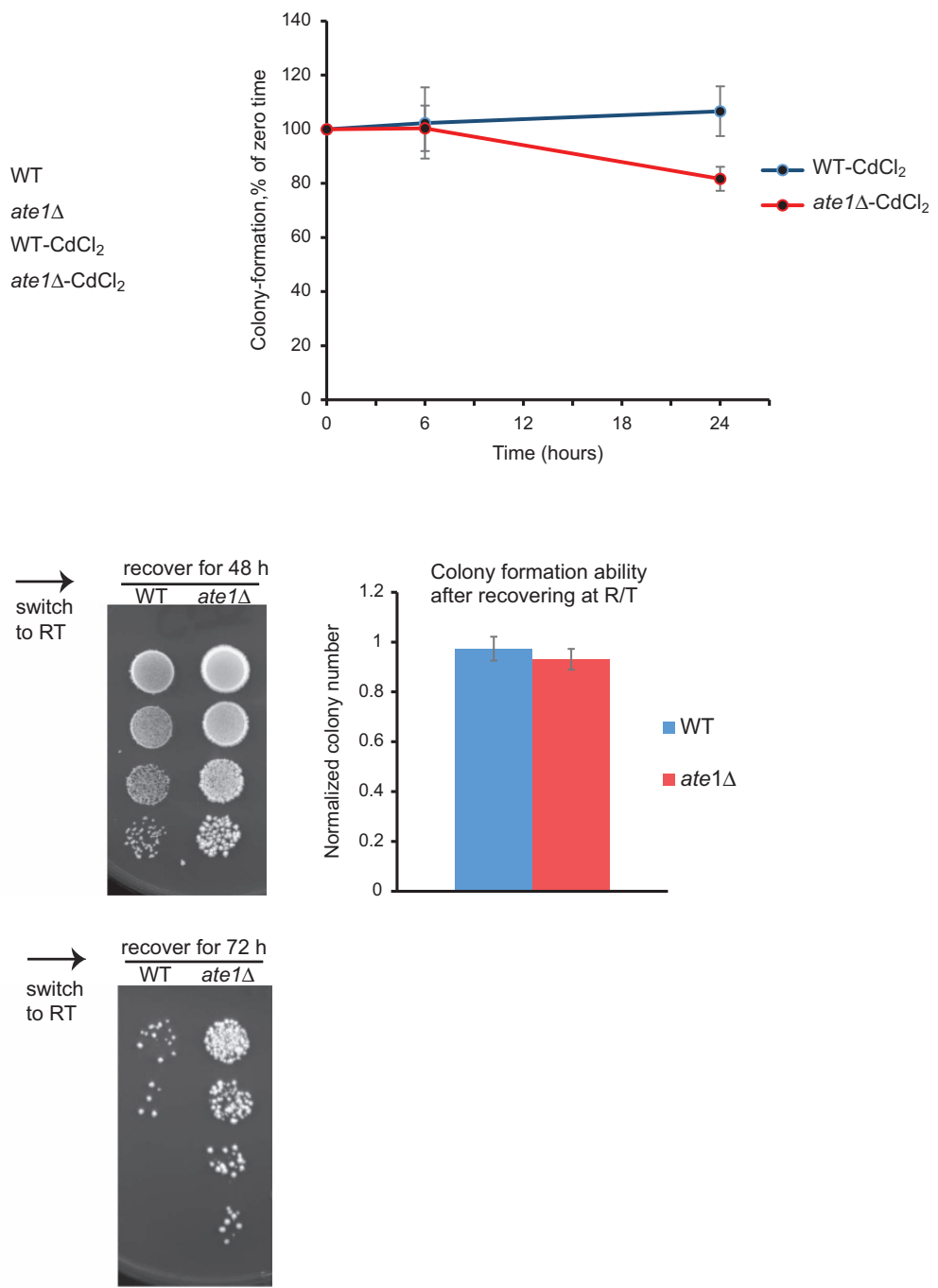
yeast strain carrying an 'in locus' 3 '-end fusion of green fluorescent protein (GFP) with the endogenous ATE1 gene (termed 'endo: Ate1-GFP') under the control of the endogenous ATE1 promoter. We found that the protein level of 'endo: Ate1-GFP' was proportionally increased with the dose of each stressors such as $\mathrm{H}_{2} \mathrm{O}_{2}$ or high salt, (Figure $4 d$ ). Similarly, when we probed the steady-state level of endogenous Ate1 in MEFs, we found a dose-dependent increase of total Ate1 protein in the presence of $\mathrm{H}_{2} \mathrm{O}_{2}$ (Figure 4f). These data suggest that Ate1 protein level is upregulated during stress in yeast and mammalian cells.

As cell death is a common outcome of extended stress response, ${ }^{36}$ we wanted to know whether the upregulation of Ate1/arginylation could be a direct inducer of cell death. To specifically test the effects of Ate1 on cell death, we employed a galactose-inducible recombinant Ate1 (termed 'pGAL1:Ate1') plasmid in yeast. To avoid interference of the endogenous Ate1, we transformed ate1 $\Delta$ yeast with this plasmid (or an empty vector as control). We found that the growth of yeast carrying the inducible Ate1 is strongly repressed on galactose-containing plate, where the expression of the recombinant protein is turned on (Figure 5a). A similar effect was observed with the induced expression of a GFP-fused Ate1 ( $p G A L$ : ATE1-GFP) (Figure 5e). To verify whether the increased abundance of Ate1 can induce cell death, yeast cells were induced in galactose-containing liquid medium for increasing durations. The cell viability was then measured by a CFU assay in glucose-containing agar plates (where the galactose induction is terminated). We found that the viability of cells carrying the $p G A L$ : ATE1-GFP was markedly decreased along the time line of galactose induction, suggesting that the upregulation of Ate1 is indeed capable of inducing cell death in yeast (Figure $5 b$ ).

To further correlate Ate 1 effects on stress response with its arginylation activity, we replaced two critical cysteine (C) residues (20 and 23) with serine (S) on Ate1. These mutations were reported to minimize the enzymatic activity of Ate1 without affecting its solubility, based on in vitro studies. ${ }^{38}$ Consistently, we found that the expression level of the C20,23S mutant Ate1 was similar to the WT version of Ate1, suggesting that these mutations did not affect the overall synthesis and turnover of this protein (Figure $5 \mathrm{c}$ ). To verify whether such mutations indeed reduce Ate 1 activity, ${ }^{37}$ we used the in-lysate arginylation assay with DD- $\beta 15-G F P$ reporter and found that the activity of the mutation is $<25 \%$ of the WT enzyme (Figure $5 \mathrm{~d}$ ). When we compared the cells overexpressing the mutant Ate1 and WT Ate1, we found that the C20,23S mutation can significantly reverse the repressing effects of Ate1 overexpression in cells (Figure 5e). Therefore, the effects of yeast Ate1 on stress response is largely (if not completely) dependent on its arginylation activity.

To test whether mAte1 has similar effects, we used splice variant 1 of mouse Ate1 (referred to as mAte1.1), the most ubiquitous isoform that exists in all tissues of animals, ${ }^{39}$ as the representative. The $\mathrm{C}$-terminal GFP-fused recombinant protein was reintroduced into ATE1-KO MEFs by a stable lowexpression system that generates recombinant proteins at a level comparable to the endogenous level of WT Ate1 (Figure $6 a) .{ }^{40}$ We found that the expression of mAte1.1 was able to reinstall cellular sensitivity to stressors, such as STS and $\mathrm{CdCl}_{2}$ (Figures $6 \mathrm{c}$ and $\mathrm{d}$ ), to levels close to WT cells, indicating that mAte1 is indeed mediating cell death in stress response. mAte1 also carries two cysteine residues (C23 and $\mathrm{C} 26$ in mAte1) corresponding to the $\mathrm{C} 20$ and $\mathrm{C} 23$ residues in yeast Ate1. Consistent with our observations in yeast Ate1, when these two cysteine residues were changed to serine in mouse Ate1, the resulted mutant (mAte1.1-mut) had compromised arginylation activity (Figure $6 \mathrm{~b}$ ), and had significantly lower capacity to reinstall cellular sensitivity to STS or $\mathrm{CdCl}_{2}$ than the original mAte1.1 (Figures $6 \mathrm{c}$ and $\mathrm{d}$ ). These data indicate that the arginylation activity of mAte1 is also required for its action in mediating stress response.

Ate1 is essential for the suppression of mutagenesis during DNA-damaging stress. Growth arrest and cell death during stress could be interpreted as a mechanism to prevent incorporation of damaged genetic material or transmission of mutation to the subsequent generations. This would raise the question whether Ate1, as a PTM enzyme, has the capacity to affect the outcome of mutagenesis.

To test whether Ate1 affects cellular response to DNAdamaging stress, we subjected cells to ultraviolet (UV) light, a common and natural mutagenic source that generates DNA damage as well as various stressing signals in cells. We found that the ATE1 deletion in yeast or MEFs significantly increased their resistance to UV light ( $254 \mathrm{~nm}$ UV-C) compared with WT (Figures $7 \mathrm{a}-\mathrm{C}$ ). In addition, the sensitivity to UV in ATE1-KO MEFs can be sufficiently reinstalled by the reintroduction of recombinant Ate1 (mAte1.1). This installation effect was nearly abolished when the catalytically impaired mutations (C23-26S) was introduced in Ate1 (Figure 7d).

To test whether Ate1 is required to prevent DNA mutations in yeast upon UV irradiation, we designed a mutation reporter Met-STOP, in which a tryptophan (TRP/W) residue in Met15 gene is replaced with an interrupting STOP codon. The

Figure 2 Knockout of ATE1 in yeast relieves growth arrest and suppresses cell death during stress response. (a) Viability of wild-type (WT) or ate1 $\Delta$ yeast after $3 \mathrm{~h}$ treatments with the indicated concentrations of the oxidative stressor $\mathrm{H}_{2} \mathrm{O}_{2}$, measured by the colony-forming unit assay and normalized to non-stressed conditions. (b) On the left are representative microscopic images showing the results of the TUNEL assay, which specifically detects DNA fragments generated during late stage of apoptosis. The WT and ate $1 \Delta$ yeast treated with $\mathrm{H}_{2} \mathrm{O}_{2} 0.5 \mathrm{mM}$. DIC images show the number of cells while the fluorescent images show cells with positive apoptosis signals (fluorescein). The graph in the right panel represents the percentage of apoptotic cells determined by TUNEL assay in WTand ate1 $1 \Delta$ yeast treated with increased concentrations of $\mathrm{H}_{2} \mathrm{O}_{2}$. (c) $\mathrm{Growth}_{\text {curves }}$ of WTand ate1 $\Delta$ yeast cultured in liquid media, in the presence or absence of the heavy metal stressor $\mathrm{CdCl}_{2}$ of $150 \mu \mathrm{M}$. Error bars represent S.E.M. ( $n=3$ ). (d) Viability of WTor ate $1 \Delta$ yeast after indicated hours of treatments with $150 \mu \mathrm{M} \mathrm{CdCl}_{2}$, measured by the colony-forming unit assay and normalized to cells at time 0 (before the application of stressor). Error bars represent S.E.M. $(n=3)$. (e) Growth test using serial dilutions of WT and ate1 $\Delta$ yeast, either in non-stressing room temperature (RT) or in high temperature $\left(40{ }^{\circ} \mathrm{C}\right)$ for $48 \mathrm{~h}$ followed by recovery at RT for another $48 \mathrm{~h}$. After recovery at RT, the numbers of colonies emerged in the lowest dilution $\left(\times 10^{2}\right)$ were quantified. The corresponding numbers for yeast constantly cultured at RT was used for normalization for WT and ate $1 \Delta$ yeast separately. Error bars represent S.E.M. ( $n=4$ for WT, $n=3$ for ate1 $1 \mathrm{)}$. (f) Growth test using serial dilutions of WTand ate $1 \Delta$ yeast, either in non-stressing RT or in high-temperature $\left(42{ }^{\circ} \mathrm{C}\right)$ for $48 \mathrm{~h}$ followed by recovery at RT for another $72 \mathrm{~h}$ 
product of this engineered gene is not expected to rescue the methionine (Met)-auxotrophy phenotype of BY4741 yeast, unless an acquired mutation reverts the interrupting STOP codon to a sense codon. (Figure $8 \mathrm{a}$ ). We found that, during non-stressing conditions, WT and ate1D BY4741 yeast carrying this reporter plasmid similarly generated negligible numbers of colonies on culture plates in the absence of methionine. However, after exposure to UV irradiations, ate1A a

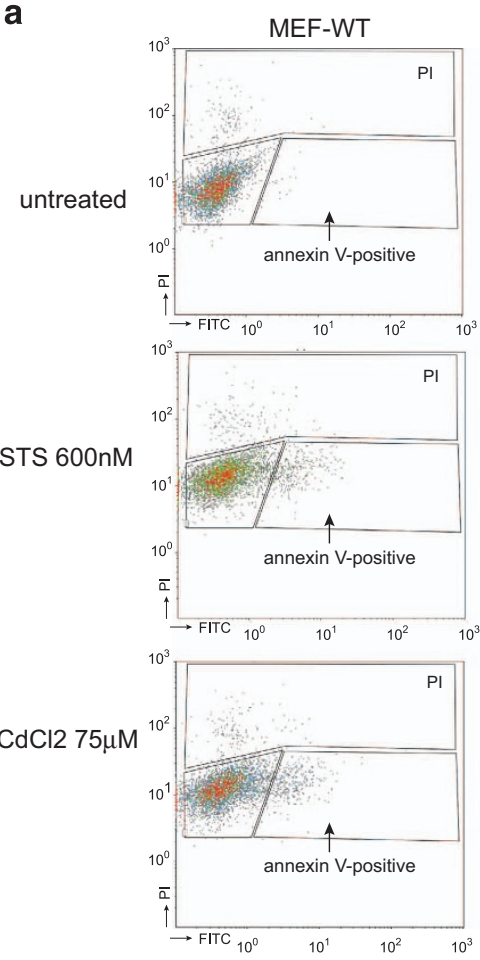

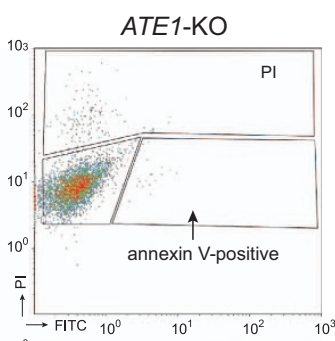
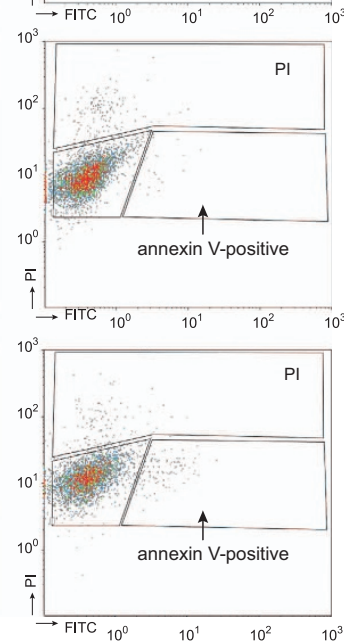
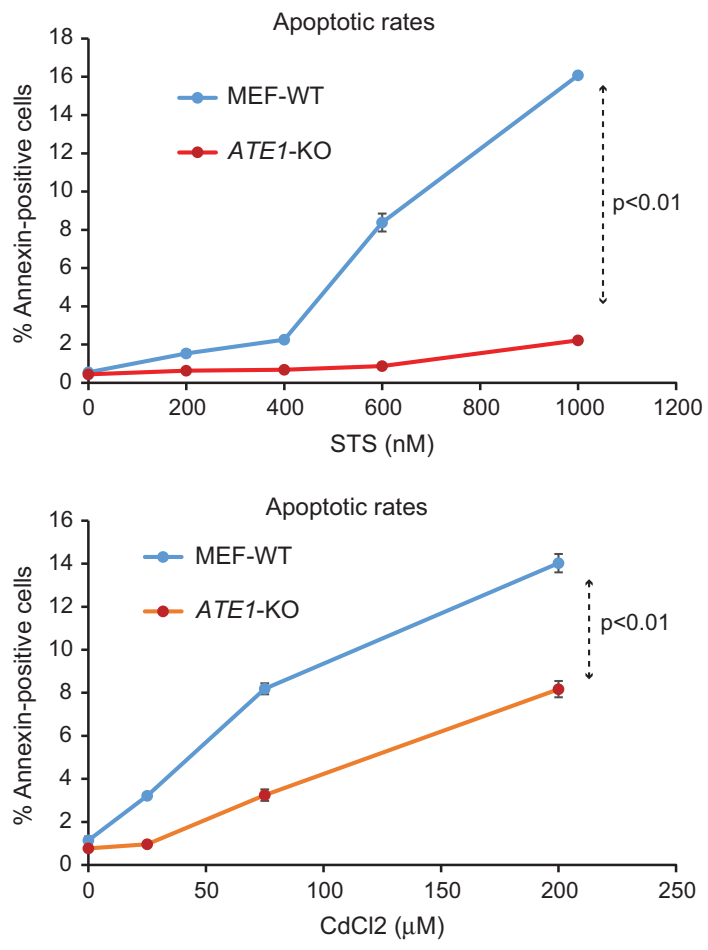

b

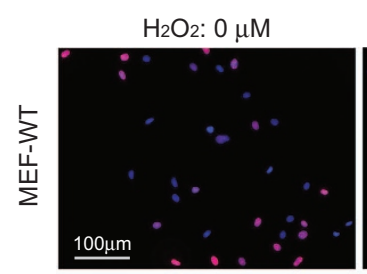

$\mathrm{H}_{2} \mathrm{O}_{2}: 300 \mu \mathrm{M}$

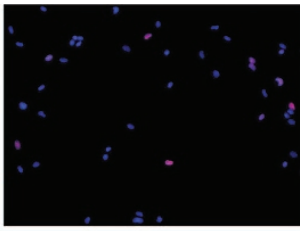

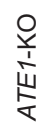
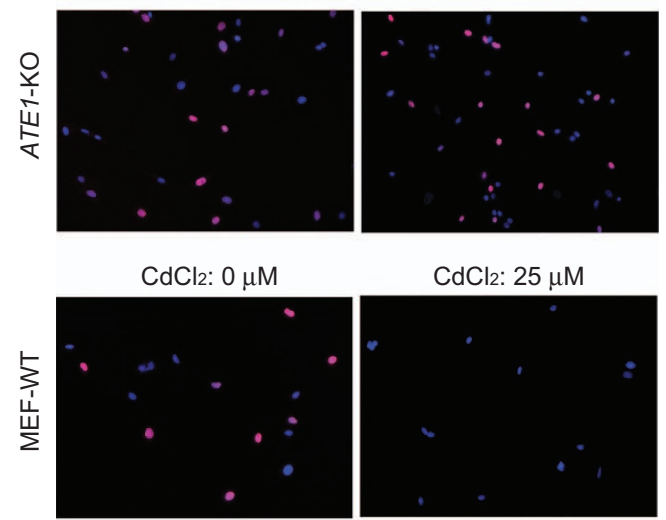

$\mathrm{CdCl}_{2}: 25 \mu \mathrm{M}$

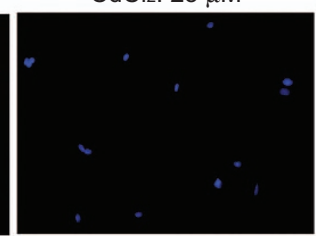

$\frac{0}{\frac{1}{4}}$
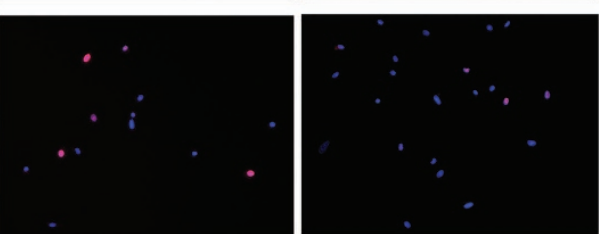

EdU (red) + Hoechst 33342 (blue)
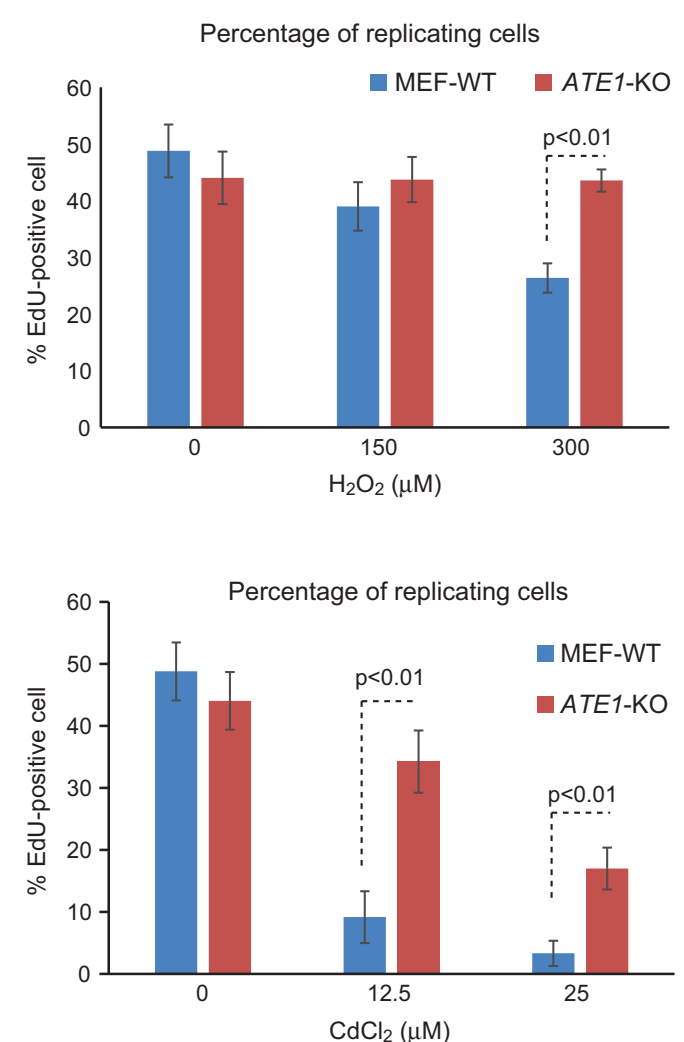
yeast produced significantly more colonies acquiring Metprototrophic phenotype compared with the WT, suggesting that the deletion of Ate1 lead to higher mutation frequencies (Figure 8b). As significant differences between WT and ate1 $\Delta$ yeast were observed in both low and high doses of UV irradiation, it is likely that both the effects of Ate1 in growth arrest and cell death contribute to the mutation suppression.

To validate these observations in mammalian cells, we created a dual-color mutation reporter system mCherryFPSTOP-IRES-GFP, in which an interrupting STOP codon replaces a Trp residue in mCherryFP gene, which is followed by an internal ribosome entry site (IRES) and a eGFP (as the internal control for transcription and expression). As such, this reporter is expected to generate a green fluorescence but not a red fluorescent signal unless a new mutation reverts the interrupting STOP codon in mCherryFP to a sense codon (Figure 8c). To measure the emergence of mutants, the WT or ATE1-KO MEFs stably transfected with the reporter, either treated with UV irradiation or not, were analyzed in fluorescence flow cytometry (FACS). In non-stressing conditions, both the WT and ATE1-KO cells had negligible numbers of red fluorescent cells. However, when exposed to a low dose of UV irradiation not expected to lead to significant cell death, a significantly higher ratio of red fluorescent cells was detected in the ATE1-KO cells compared with the WT cells (Figures $8 \mathrm{~d}$ and e). To further validate that the red fluorescence detected by FACS was genuine, the positive cells were sorted and subsequently examined by microscopy. We found that the majority of collected cells exhibited both red and green fluorescence, with $<5 \%$ being false positives having only green fluorescence (Figure 8f). Finally, to confirm the mutation in the reporter gene, we sequenced the corresponding region with genomic DNA prepared from these red-positive cells and identified a reverting mutation in the anticipated location, which was absent in the untreated cells (Supplementary Figure S2).

\section{Discussion}

Although arginylation has been known for more than 50 years, the mechanism underlying the physiological roles of this PTM at the cellular, tissue and organismal levels remain undefined. Our study reveals that Ate1 and Ate1-mediated arginylation are upregulated as part of the general stress response to induce cell growth arrest and cell death, which results in the prevention of DNA mutagenesis under stress. Our data therefore clarify the nature of the involvements of Ate1 and its arginylation activity in stress response, potentially providing a novel explanation for the in vivo functions of Ate1 and arginylation in a host of human diseases.
Our data indicate that Ate1 and arginylation have a proapoptotic role, which appears to contradict several studies suggesting an anti-apoptotic role. ${ }^{23-28}$ This discrepancy may be explained by several factors. First, many previous studies focused on individual arginylation substrates. Although those results may still be relevant to the particular pathway studied, they may not be representative of the role of Ate1 or arginylation at the global level. Second, as our data pointed out, Ate1 and arginylation can lead to either growth arrest or cell death, depending on the nature and intensity of the stressor. The growth arrest may occasionally render effects closely resembling anti-apoptotic events. However, our data clearly demonstrate that in the presence of a lethal stressor, Ate1 and arginylation have a pro-apoptotic role.

Our study has unequivocally shown that Ate1 and arginylation are essential for physiological response to a variety of stress conditions including heat, oxidative and osmotic stresses, as well as exposure to heavy metal and radiation. Although there is a certain degree of species variation, overall, a downregulation of Ate1 leads to an increased stress resistance. It is worth pointing out that many of these stressing conditions are known to have causative effects for human diseases such as cancer, cardiovascular disease, aging, developmental abnormalities, injury and inflammation. Interestingly, biochemical and genetic evidence have shown a fluctuation of arginylation activity or disruption of ATE1 gene being correlated with some of these diseases. ${ }^{2,3,9-18,41-44}$ Thus, our results thus have the potential to create new therapeutic interventions based on Ate1/arginylation for these diseases. In particular, our results provide a plausible explanation for the observed but poorly explained correlation between the downregulation of Ate1/arginylation and the increased risk of tumorigenesis, metastasis and radiotherapy resistance. ${ }^{41,42,45,46}$ Tumor microenvironments are often highly stressing due to poor formation of blood vessels or high activity of mitogenic factors. Based on our data, a downregulation of Ate1/arginylation would assist cancer cells to survive the stress, and to accumulate mutations, thereby increasing tumor growth and metastatic risks. These interesting possibilities require further explorations.

Several questions remain open following our study. The complete mechanistic connection between Ate1/arginylation and stress response still awaits elucidation. Also, how Ate 1 is being regulated on expression level in normal and transformed cells requires further investigation. In addition, although our data suggest that the effects of Ate1 is largely dependent on its arginylation activity, we cannot dismiss possible involvement of potential regulatory proteins. ${ }^{37,47}$

Figure 3 Knockout of ATE1 in MEFs results in attenuated apoptosis and growth arrest during stress response. (a) Annexin-V-Alexa Fluor 488 (Thermo Fisher) was used to detect phosphatidylserine inversion on the peripheral membrane in an early stage of apoptosis. WT or ATE1-KO MEFs were treated with STS or CdCl ${ }_{2}$ of different concentrations for $5 \mathrm{~h}$. Propidium iodide (PI) was used to label necrotic and late apoptotic fractions. The cell population is analyzed by FACS. On the left panel representative FACS charts are shown. The gate settings for Annexin-V and PI detection are indicated. Quantification of data from three independent repeats $(n=3)$ are shown in graphs presented on the right side. ATE1-KO cells have a much lower apoptotic rate than WT cells. No obvious difference in PI staining was found in the conditions we tested. (b) On the left panel are the representative microscopic images showing WT and ATE1-KO MEFs treated with $\mathrm{H}_{2} \mathrm{O}_{2} 0.5 \mathrm{mM}$. EdU (red fluorescently labeled), which is incorporated into newly synthesized DNA during the $S$ phase of cell cycle, was used as a marker for cell proliferation. Hoechst 33342 (blue) was used to show the number and morphology of nucleus. Only cells with normal nuclear morphology and no sign of apoptotic bodies were included for quantification. At least 10 randomly chosen images in each group were used to generate the graphs shown on the right side for quantification of active replicating cells with EdU incorporated into their nucleus, in the presence of stressors $\mathrm{H}_{2} \mathrm{O}_{2}$ and $\mathrm{CdCl}_{2}$. Error bars represent S.E.M. 
a

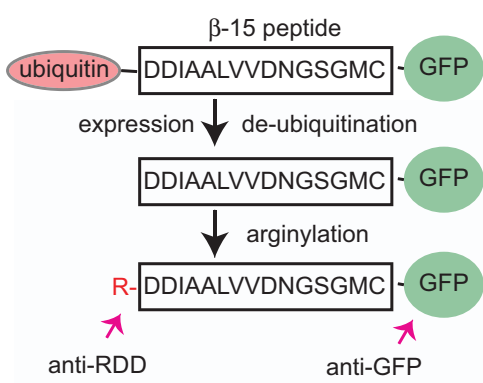

C

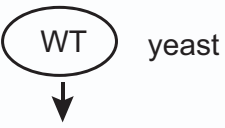

Stress for different duration

$$
\downarrow
$$

cell lysate $+\mathrm{DD}-\beta$-15 peptide. GFP

$$
\downarrow
$$

in-lysate arginylation reaction for 10 minutes

$$
\downarrow
$$

probed with anti-RDD and anti-GFP

\section{b}
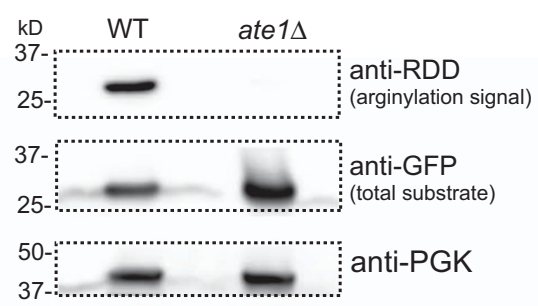

d

endo:Ate1-GFP:

on yeast chromosome VII

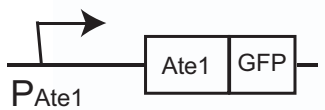

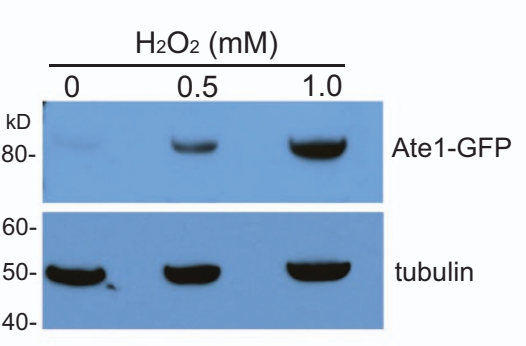

e in-lysate arginylation of MEF
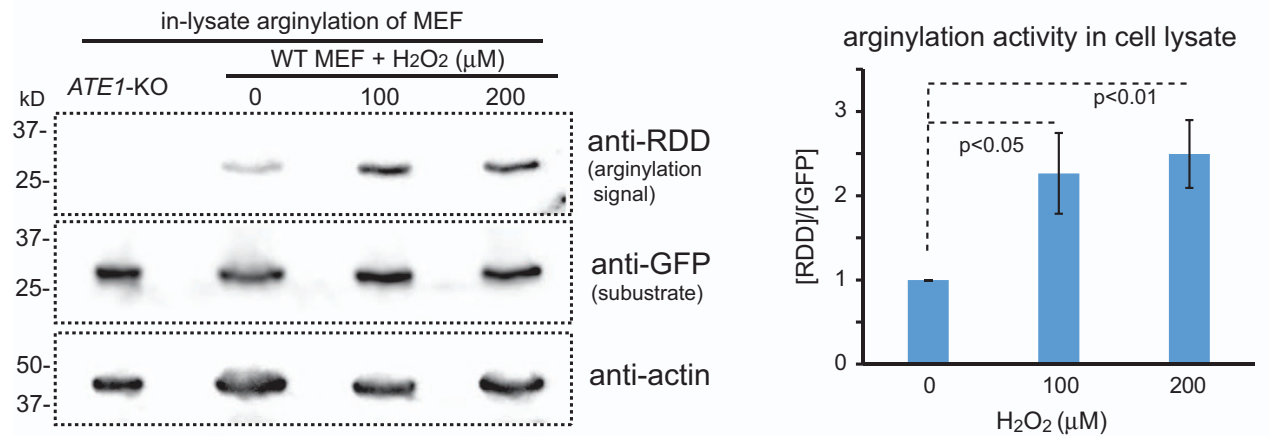

f
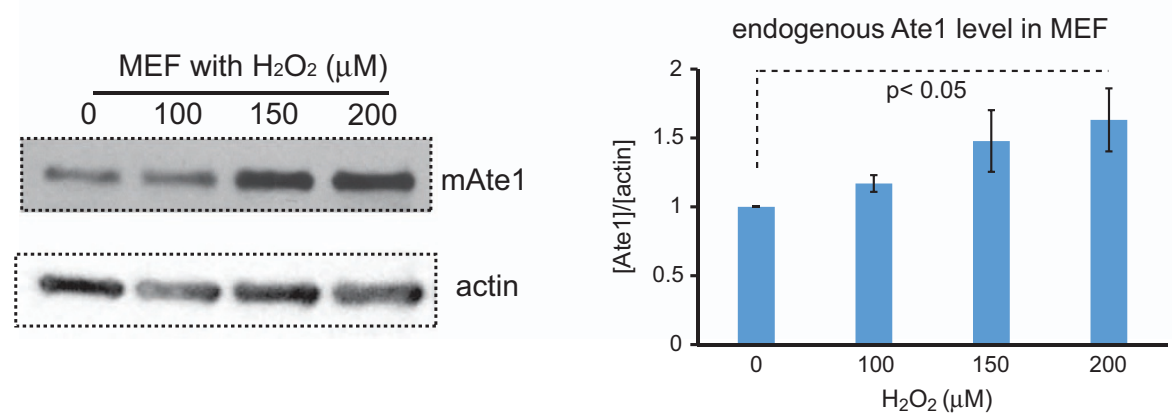
Finally, although the actions of other PTM (such as acetylation or phosphorylation) on certain proteins may also affect genetic fidelity, ${ }^{48,49}$ the global consequence of those modifications have never been demonstrated. Our study is the first example showing that arginylation is a PTM with a unique global effect on mutagenesis.

\section{Materials and Methods}

Yeast strains. The $S$. cerevisiae strains used in this study are parental WT

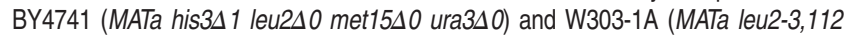
trp1-1 can1-100 ura3-1 ade2-1 his3-11,15 ybp1-1), both obtained from Open Biosystems (Lafayette, CO, USA). A strain carrying a null ate1 $1 \Delta$ KanMX cassette in the BY4741 background was obtained from Open Biosystems and the cassette was subsequently transferred to the W303-1A background using Ate1-300UP and Ate1300Down primers. The strain with a GFP fused to the C-terminal end of endogenous Ate1 in the native chromosome locus in BY4741 genetic background was obtained from Open Biosystems. The identity of each $\mathrm{KO}$ strain listed above was confirmed with PCR genotyping.

Culture of yeast. Yeast culture media were prepared as described below:

YPD: $2 \%$ glucose, $1 \%$ yeast extract and $2 \%$ peptone. SD (synthetic defined) medium (per $1000 \mathrm{ml}$ ): yeast nitrogen base $(1.7 \mathrm{~g})$, ammonium sulfate $(5 \mathrm{~g})$, dextrose/galactose/raffinose $(20 \mathrm{~g})$, required amino acids $(50 \mathrm{mg})$ and uracil (if required; $50 \mathrm{mg}$ ).

For solid media plates, $2 \%$ agar was added to the liquid media.

Strains grown in liquid and solid media were incubated at $30^{\circ} \mathrm{C}$ unless otherwise indicated.

For most serial dilution growth assays, a single colony of yeast was inoculated in liquid medium and allowed to grow to the log phase before spot plating of serial dilutions as described elsewhere. ${ }^{50}$

Mammalian cells and media. Immortalized MEFs carrying either WT ATE1 or KO for ATE1 (ATE1-KO) were a gift from Dr. Anna Kashina (University of Pennsylvania), prepared as described elsewhere. ${ }^{33}$ Immortalized HFFs were a gift from Dr. John Murray (University of Indiana). A human embryonic kidney cell line (HEK 293 T; clone T7) was obtained from ATCC.

Mammalian cells were grown in media containing a 1:1 mix of DMEM high glucose and F10 media (Life Technologies, Grand Island, NY, USA, Cat\# 11995-065 and 11550-043) with 10\% FBS (HyClone, Pittsburgh, PA, USA, Cat\# SH30910.03), supplemented with antibiotic-antimycotic (Life Technologies, Cat\# 15240-096). The cells were cultured in a $5 \% \mathrm{CO}_{2}$ incubator at $37^{\circ} \mathrm{C}$, unless otherwise indicated. The MEF cells are continuously split in every 3 days to keep them in active growing stages. Old cells that pass 24 passages were discarded and younger cells were revived from frozen stock. Also, to avoid the interference of contact inhibition, only cells with culture density of $<50 \%$ were used for any experiments in this study, unless otherwise indicated.

Molecular cloning and preparation of plasmids. All molecular cloning work was performed with high-fidelity DNA polymerase Herculase (Agilent, Santa Clara, CA, USA). Ligation products were transformed into chemical competent E. coli TOP10 (Life Technologies).

PCR reactions were performed in either a Veriti Thermo Cycler (Applied Biosystems, Grand Island, NY, USA) or a T100 Thermo Cycler (Bio-Rad, Hercules, CA, USA). Most primers were ordered from IDT (Coralville, IA, USA) or Sigma (St. Louis, MO, USA).

Primers used for the creation of ATE1 deletion in yeast with the cassette of ate $1 \Delta$ : KanMX

ATE1300UP: ATGGTGCTGTGCTTGTAATTG CC

ATE1300DOWN: GCTCATCAAAAACTAAGAATAAGAG

The yeast Ate1 overexpression construct was cloned for insertion into the galactose-inducible pYES2 yeast expression vector containing a GAL1 promoter (Life Technologies, \# V825-20) at the Kpn1 and Xba1 sites using the following primers:

yATE, forward: TACTGGTACCGCCATGTCCGATAGATTCGTTATTTGGGC

yATE, reverse: TAATTCTAGATCACATTTGCTCACTATATAAAATGACGGC

pGAL: ATE1-GFP cloning: for in-frame fusion of ATE1 with GFP coding sequence, the stop codon of ATE1 was removed. Then, a TEV cleavage site linker (ENLYFQGTGGIHRPVAT) was added between Ate1 and GFP, as described before. ${ }^{37}$ The coding sequence for $p G A L$ : Ate1-GFP fusion protein was constructed using the following primers for insertion into the PYES2 vector:

yATE-Xho1R: GTATCGTCTAGAGGCGCCCTCGAGCATTTGCTCACTATATAAAATGAC yATE-GFP, forward: GTATCGCTCGAGGAAAACCTGTATTTTCAGGGAACCGG yATE-GFP, reverse: GTATCGTCTAGATCACTTGTACAGCTCGTCCATGCCGAGAG

Ate1 point mutations: point mutations of yeast Ate1 for C20,23S were created using the following primers:

yATE-20,23SSF: CAATGAACCTGCCGCAAAGTCTGGGTATTCTCACGGTAATAA GGGGGGC

yATE-20,23SSR: GCCCCCCTTATTACCGTGAgAATACCCAgACTTTGCGGCAGG TTCATTG.

DD- $\beta 15$-GFP, arginylation reporter cloning: the CDNA of the arginylation reporter contains in-frame fusion of the ubiqutin gene on its $5^{\prime}$-region, followed by the coding region of 15 amino acid codons of $\beta$-actin starting at the third residue (DDIAALVVDNGSGMC), a linker region, and then a coding region for C-terminal GFP. It was cloned in a yeast expression vector, pGPD2 (Addgene, Cambridge, MA, USA, \# 43972), at EcoR1 and Xho1 sites. A plasmid containing the sequence of ubiquitin-DD- $\beta$-actin was used as a template, as reported. ${ }^{51}$ The following primers were used for this cloning:

Beta-ReporterF: GATAAAGAATTCATGCAAATTTTCGTCAAGAC

Beta-ReporterR: GTCGACCTCGAGTTACTTGTACAGCTCGTCCATG

\footnotetext{
Figure 4 The levels of Ate1 protein and global arginylation activity are upregulated during stress. (a) A scheme illustrating how DD- $\beta 15$-GFP is used as the reporter of arginylation activity. The fusion protein containing a stretch of 15 amino acids starting with two aspartic acids (D) derived from the $\mathrm{N}$ terminus of mammalian $\beta$-actin, a known substrate of arginylation. ${ }^{31}$ This peptide is fused with an $\mathrm{N}$-terminal ubiquitin, which is cleaved co-translationally by endogenous de-ubiquitylation enzymes in eukaryotic system and leaves the aspartic acids as the new N terminus. The arginylation state of this reporter can be probed with an anti-RDD antibody, which only reacts with the arginylated form of the reporter protein. A C-terminal GFP tag is used to facilitate the detection of steady-state level of the reporter protein by immunoblotting with anti-GFP antibody. (b) The arginylation level of DD- $\beta 15$-GFP expressed in either WT or ate $1 \Delta$ yeast was examined with anti-RDD antibody, which only shows a visible signal in the WT cells. An antibody for GFP was used to probe the total protein level of the DD- $\beta 15-$ GFP. PGK was used as a loading control for total yeast cellular proteins. (c) lllustrative scheme (left panel) and representative immunoblots (right panel) showing the arginylation activity in cell lysates prepared from yeast exposed to $1 \mathrm{M} \mathrm{NaCl}$ stress for increasing times. The lysates were then mixed with the recombinant protein DD- $\beta 15$-GFP prepared from ate $1 \Delta$ yeast for an in-lysate arginylation reaction for 10 min at RT. The arginylation level of the reporter protein was detected by immunoblotting with anti-RDD antibody. The steady-state level of the reporter protein was probed with anti-GFP. An anti-3-phosphoglycerate kinase (PGK) antibody was also used as a loading control. (d) On the left, a scheme illustrating the domain structure of the 'in locus' GFP-fused Ate1, which is driven be the endogenous ATE1 promoter at the native chromosome locus (Chromosome VII) in the yeast (termed 'endo: Ate1-GFP'). The right panels present immunoblots showing the steady-state levels of 'endo: Ate1-GFP' in yeast treated with increasing concentrations of different stressors: $\mathrm{H}_{2} \mathrm{O}_{2}$ (left) or $\mathrm{NaCl}$ (right). Tubulin or PGK was used as loading controls. (e) WT MEFs were exposed to increased concentrations of $\mathrm{H}_{2} \mathrm{O}_{2}$ for $30 \mathrm{~h}$. The lysates from all these cells, as well as untreated ATE1-KO MEFs (as a control), were then mixed with the recombinant protein DD- $\beta 15$-GFP purified from ate $1 \Delta$ yeast for an in-lysate arginylation reaction for $45 \mathrm{~min}$ at RT. The arginylation level of the reporter protein was detected by immunoblotting with anti-RDD antibody. The steady-state level of the reporter protein was probed with anti-GFP. Actin antibody was used as a loading control. The graph on the right side shows quantification from four independent repeats. (f) Left: representative immunoblots showing the levels of endogenous Ate1 proteins in MEFs treated with increasing concentrations of $\mathrm{H}_{2} \mathrm{O}_{2}$ for $30 \mathrm{~h}$, detected by a specific antibody for mouse Ate1 (Millipore, clone 6F11) recognizing all four major Ate1-splicing variants. Actin was used as loading controls. Right: quantification of the endogenous Ate1 level in $\mathrm{MEFs}$ treated with $\mathrm{H}_{2} \mathrm{O}_{2}$ from three independent repeats. The Ate1 level was calculated by normalization with actin loading control, and then further normalized to the level at non-stressing condition $\left(0 \mu \mathrm{M} \mathrm{H}_{2} \mathrm{O}_{2}\right)$. In all above figures, error bars represent S.E.M. and statistical significance was calculated by Student's t-test
} 


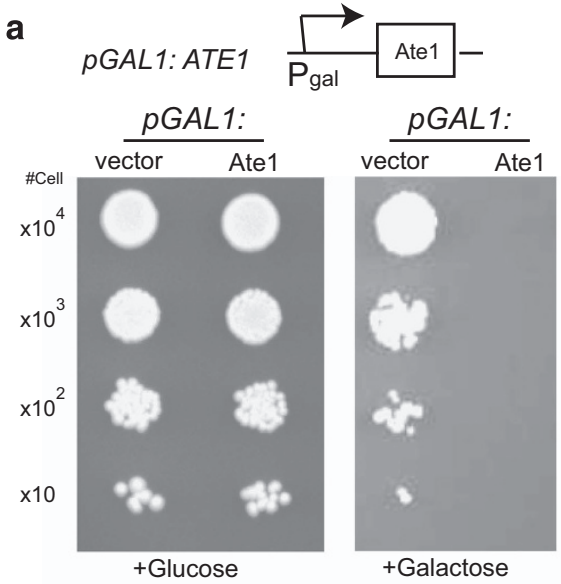

C

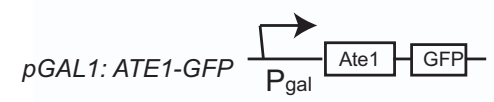

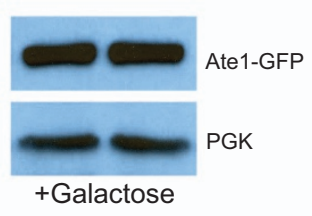

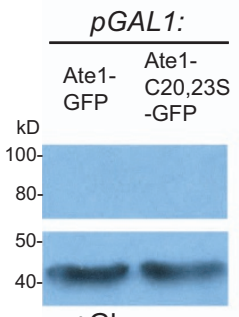

+Glucose

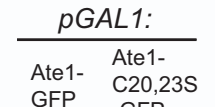
GFP -GFP

e

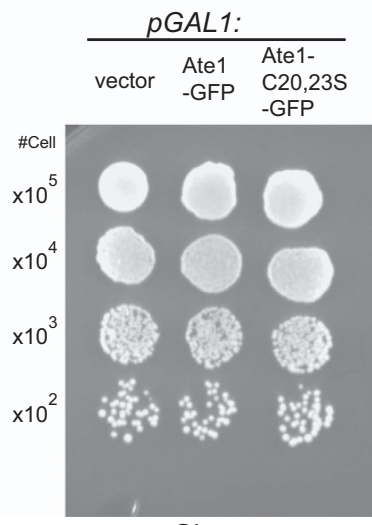

+Glucose b Viability of cells after galactose-induced over-expression of Ate1

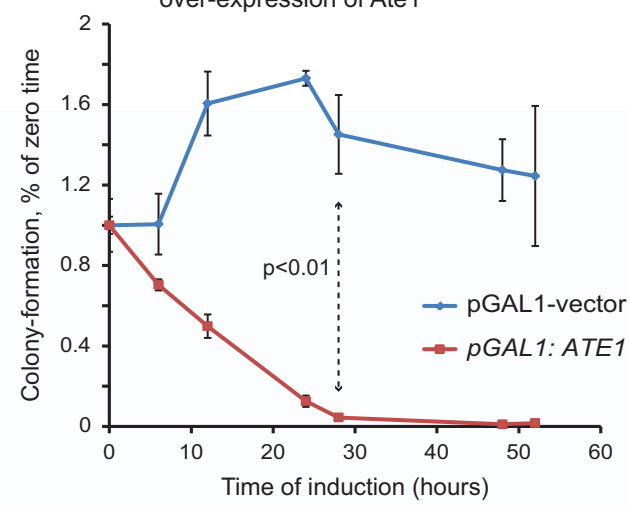

d

pGAL1-Ate1-GFP or Ate1-C20,23S-GFP

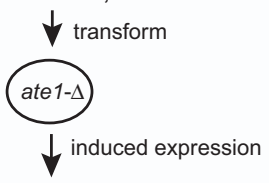
cell lysate + DD- $\beta 15-G F P$
$\downarrow$

in-lysate arginylation reaction

$\downarrow$

probed with anti-RDD and anti-GFP

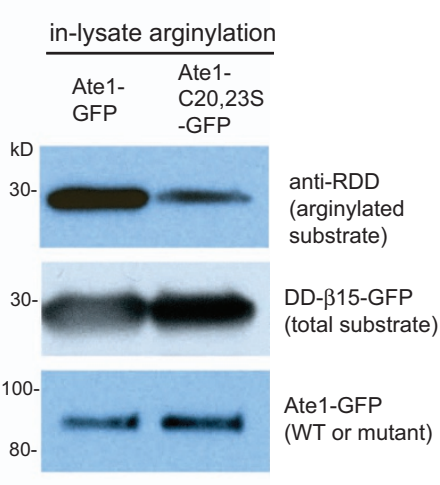

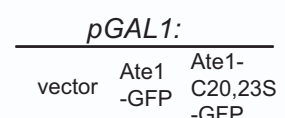

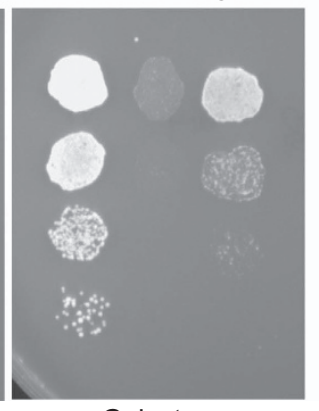

+Galactose

Figure 5 The increase of Ate1 triggers cell death in yeast in a manner that is dependent on its arginylation activity. (a) The scheme in the top panel shows the domain structure of plasmid pGAL1: ATE1, in which the coding sequence of recombinant protein is preceded by the inducible GAL1 promoter. The picture in the bottom panel shows the growth of ate1 $\Delta$ yeast cells carrying either the empty expression vector or $p G A L 1$ : ATE1 by a serial dilution growth assay on either plate containing glucose (suppressing) or galactose (inducing). (b) Graph showing the viabilities of ate1 $1 \Delta$ yeast cells carrying either the empty expression vector or pGAL1: ATE1 in different time points following the initiation of galactose-induced expression, as measured by the numbers of colony-forming cells per OD unit (CFU) that were normalized to starting data point time 0 , for Ate 1 or vector control separately. Error bar represents S.E.M. $(n \geqslant 3)$. (c) The top scheme shows the domain structure of a recombinant Ate1 fused with a linker and a C-terminal GFP, driven by the pGAL1 promoter, termed ' $p G A L$ : Ate1-GFP'. Immunoblot analysis of the steady-state levels of wild-type and C20,23S mutant Ate1 after 9-h culture in the presence of non-inducing (glucose) or inducing (galactose) carbon sources. PGK was used as loading controls. Anti-GFP was used to probe the steady-state levels of the recombinant ' $p G A L$ : Ate1-GFP' (WTor mutant). (d) Left panel showing the procedure of using an in-lysate arginylation assay to measure the activities of recombinant Ate1-GFP, either the WT version or the $\mathrm{C} 20,23 \mathrm{~S}$ mutant, which were expressed for $9 \mathrm{~h}$ in ate1 $\Delta$ yeast (see $\mathbf{c}$ for the steady-state levels of expressed proteins). Anti-RDD was used to indicate the level of arginylated reporter. Anti-GFP was used to show the total amount of reporter protein (DD- $\beta 15-G F P$ ) in each sample, as well as the total amount of Ate1-GFP (either WT or mutant) present in each sample. These two bands were distinguished by their difference in molecular weight (27 kDa versus $92 \mathrm{kDa})$. (e) Representative pictures of growth test using serial dilutions of ate1 1 yeast carrying either the empty expression vector or pGAL1-Ate1-GFP, or pGAL1-Ate1-C20,23S-GFP, in media containing non-inducing (glucose) or inducing (galactose) carbon sources 
a

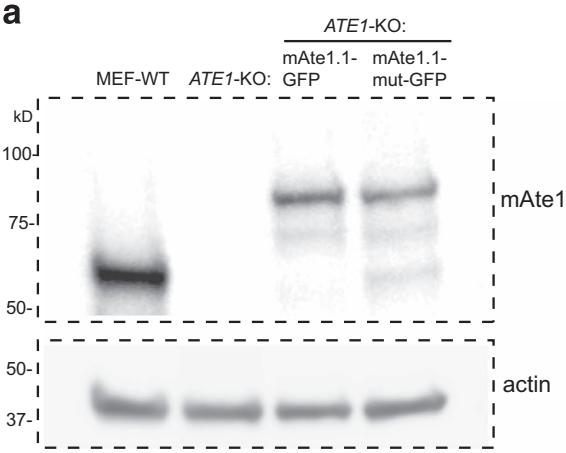

b

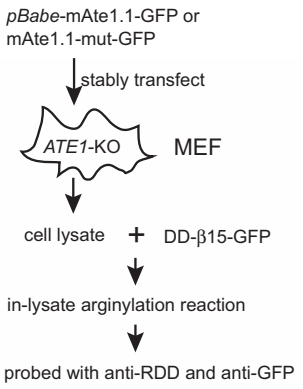

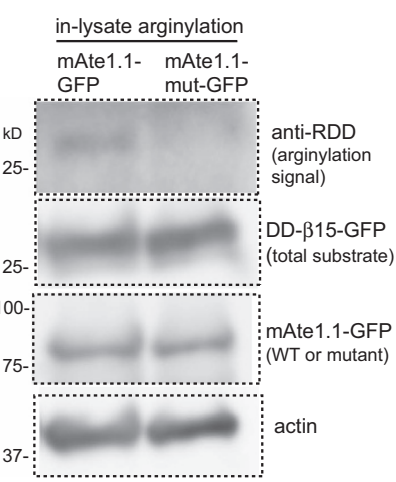

d Viable cell numbers after treatment of $\mathrm{CdCl}_{2}$

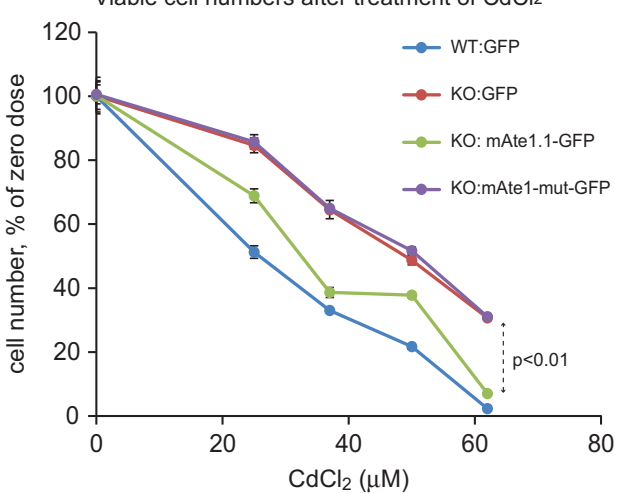

Figure 6 Mammalian Ate1 is required for cellular sensitivity to stressors in a manner dependent on its arginylation activity. (a) Representative immunoblots showing the levels of Ate1 protein detected by a specific mouse Ate1 antibody (Millipore, clone 6F11) as either endogenous Ate1 in WT MEF or the recombinant mouse Ate1-isofrom 1 (WTor C23$26 \mathrm{~S}$ mutant) fused with a C-terminal GFP stably transfected in ATE1-KO cells. Actin was used as a loading control. (b) Left panel showing the procedure of using an in-lysate arginylation assay to measure the activities of either the WT version of mAte1.1-GFP or the mutant with cysteine 23 and 26 to serine replacement (referred as mAte1.1-mut-GFP in this study) expressed in stably transformed ATE1-KO MEF. The arginylation reporter protein, DD- $\beta 15-G F P$, as described in Figure 4 , was expressed and purified from ate $1 \Delta$ yeast. On the right panel, anti-RDD was used to probe the level of arginylation on the reporter protein. Anti-GFP was used to show the total amount of reporter protein (DD- $\beta 15$ GFP) added in each sample. Mammalian Ate1 antibody (Millipore, clone\# 6F11) was used to detect the level of mAte1.1-GFP (either WTor mutant) present in each sample. Actin was used as a loading control for cell lysates. (c) Graph showing the quantification of cell viability after treatment of different concentrations of STS for $12 \mathrm{~h}$, for WT and ATE1-KO cells stably expressing either GFP (as transfection and expression control), GFP-fused recombinant mouse Ate1.1 or enzymatically impaired mutant mouse Ate1.1. The viable cells were counted by cell counter and using Trypan blue to exclude dead cells. For each group, the data were normalized to a sample under non-stressing condition. Error bars represent S.E.M. $(n=3)$. (d) Similar to (c), except that a treatment of $\mathrm{CdCl}_{2}$ was used. Error bars represent S.E.M. $(n=3)$

pHLUM-Met-STOP cloning: a nonsense mutation was created in the MET15 gene of pHLUM plasmid (Addgene, \# 40276) to change nucleotide number 954 from $\mathrm{G}$ to A, which converts the Trp318 codon to a STOP codon. For this purpose the following primers were used:

pHLUM-metstop-SnaBF: TCCCCATACGTATCTTGAGTTTCATACCCTGG (containing the mutation site)

pHLUM-metstop-XhoR: GTCTCCCTCGAGCTTGTGAGAGAAAGTAGG

PCR product from the above primers were then digested with restriction enzymes SnaBl and Xhol, and ligated back to the vector that was digested with the same enzymes.

mCherryFP-STOP cloning: a nonsense mutation was created in the mCherryFPcoding sequence to change nucleotide number 294 from $G$ to $A$, which converts the Trp98 residue to a STOP codon. For this purpose, the following primers were used:

mCherry-Stop-F: CCCCGAGGGCTTCAAGTGAGAGCGCGTGATGAACTTCG mCherry-Stop-R: CGAAGTTCATCACGCGCTCTCACTTGAAGCCCTCGGGG

The above primers were used in an overlapping PCR in combination with a pair of cloning primers upstream and downstream of the mCherryFP gene:

mCherry-Notl-F: TAT ATA TGC GGC CGC ATG GTG AGC AAG GGC GAG GAG G

mCherry-TAA-BamHI: ATATGGATCCTTACTTGTACAGCTCGTCCATGCCGCC

The final product was inserted by restriction sites of Notl and BamHI before the IRES region in the PQC-XIG vector (Addgene, w497-1).
pBabe-puro vectors containing eGFP or mAte1.1-eGFP were gifts from Dr. Anna Kashina (UPenn). The preparation of those vectors was described elsewhere. ${ }^{40}$ The primers for creating $\mathrm{C} 23,26 \mathrm{~S}$ mutations on mAte1.1 are:

Forward mutant primer: GCCAGACCTCCTTCCAGaGcGGCTACaGCAAGAACA AGTTGGGCAGTCG

Reverse mutant primer: CGACTGCCCAACTTGTTCTTGCtGTAGCCgCtCTGGA AGGAGGTCTGGC

The below two primers pairs were used with the above primers for overlapping PCR with the original $p B a b e-m A t e 1.1-e G F P$ as template:

Psi-Spel: GCCTGCGTCGGTACTAGTTAG

Ate1-EcoRI: GAATGAATTCTTTAGACCCTTC

The final product was digested with Spel and EcoRI, and then ligated into the pBabe-mAte1.1-eGFP that was cut with the same enzymes.

Preparation of protein sample and analysis with SDS-PAGE and western blot. For yeast, the cells were lysed by vortex with glass beads with $1 \times$ Laemmli SDS-loading buffer. The lysate was then heated in a boiling-water bath for $10 \mathrm{~min}$. For mammalian cells, cells were first briefly washed with phosphatebuffered saline (PBS) and then spun down at $2400 \times g$ in a desktop centrifuge. The cell pellet was then weighed and lysed with five volumes of PBS and five volumes of $4 \times$ Laemmli SDS-loading buffer and boiled for $10 \mathrm{~min}$, as described. ${ }^{51}$

The protein samples were separated on SDS-PAGE and then transferred to the nitrocellulose membrane for wWestern blot analysis. For GFP or GFP fusion 


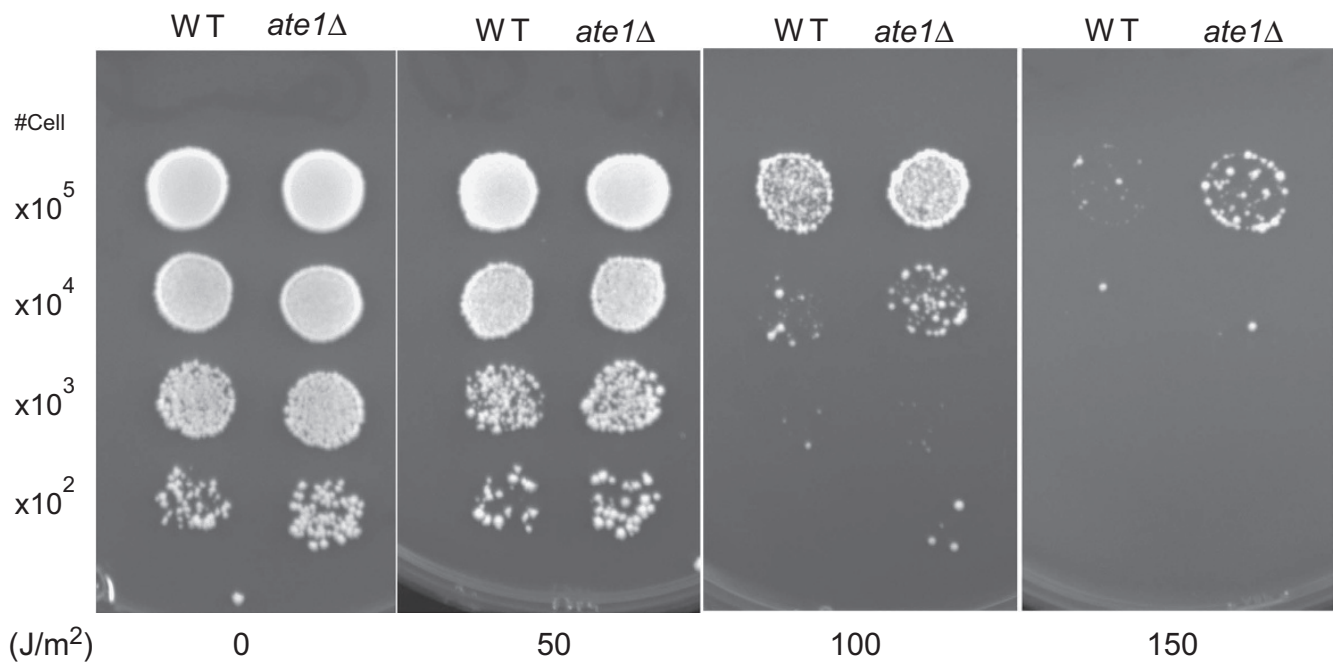

b UV dose $\left(\mathrm{J} / \mathrm{m}^{2}\right)$

50

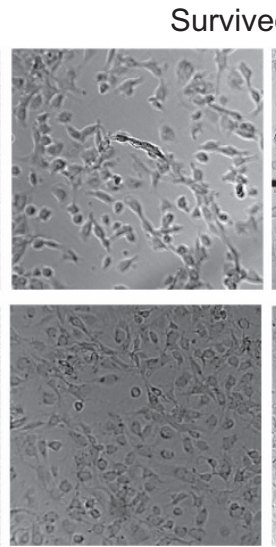

75
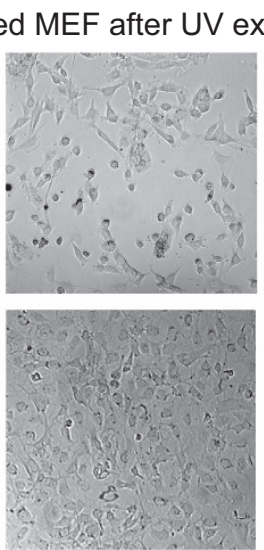

100
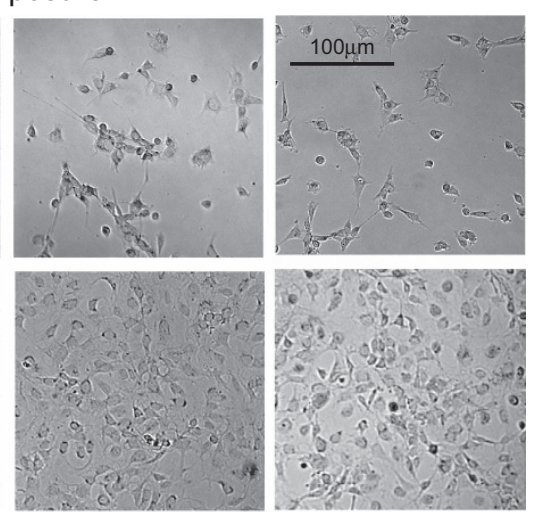

200

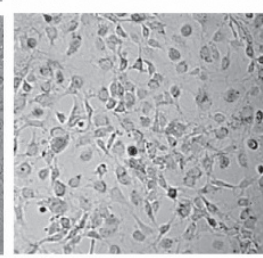

400 c

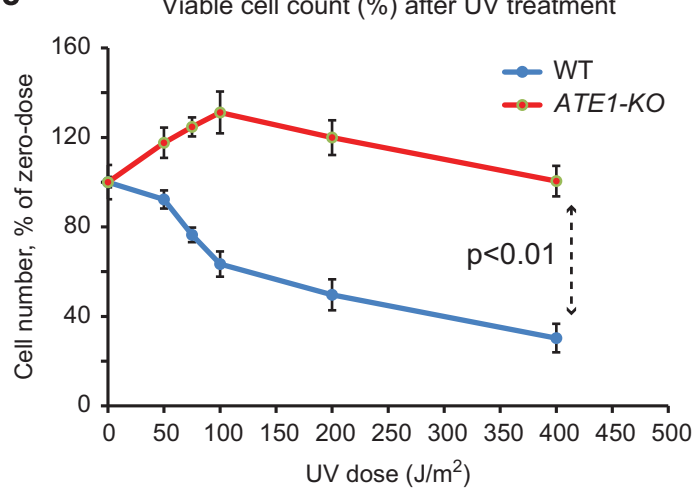

d Viable cell count (\%) after UV treatment

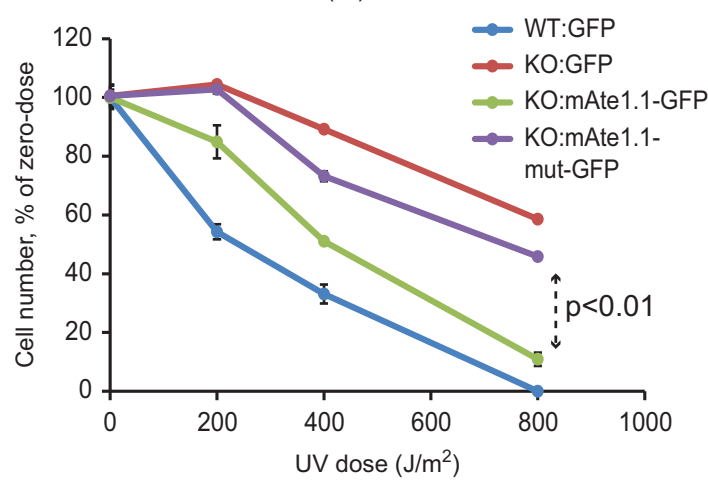

Figure 7 Knockout of ATE1 increases cell viability upon UV irradiation. (a) Representative images of WTand ate $1 \Delta$ yeast grown for 3 days after exposure to different doses of UV irradiation and recovery in the dark. (b) Representative images showing WT or ATE1-KO MEFs after exposure to increasing doses of UV irradiation and recovery for $24 \mathrm{~h}$. (c) Quantification of viable cells at $12 \mathrm{~h}$ after UV treatment. Live cells were quantified with cell viability dye Calcein $\mathrm{AM}$ and the numbers were normalized to matching samples not irradiated $\left(0 \mathrm{~J} / \mathrm{m}^{2}\right)$. Error bar represents S.E.M. $(n=3)$. (d) Comparison of cell viabilities at $12 \mathrm{~h}$ after UV treatment for WT or ATE1-KO cells stably expressing either GFP (as transfection and expression control), mAte1.1-GFP or the catalytically impaired mAte1.1-mut-GFP. The number of viable cells was directly counted by cell counter and using Trypan blue to exclude dead cells 
proteins, monoclonal mouse anti-GFP (Roche, Branchburg, NJ, USA, clone 7.1 and 13.1, Cat\# 11814460001) was used. For mouse or human Ate1, a monoclonal rat anti-Ate1 (Millipore, Billerica, MA, USA, clone \# 6F11, Cat\# MABS436) was used. For loading controls, anti-yeast-tubulin (Abcam, Cambridge, MA, USA, \# ab6161), antiyeast-GAPDH (Thermo Fisher Scientific, Grand Island, NY, USA, \#MA5-15738), antiyeast-phosphoglycerate kinase (PGK; Life Technologies \#459250) and anti-betaactin (Sigma, clone AC-15, Cat \#A1978) were used. HRP-conjugated secondary antibodies, including anti-mouse/rabbit-HRP (Roche) and anti-rat-HRP (Santa Cruz, Dallas, TX, USA) were used with a chemiluminescence kit (Roche) to visualize the protein bands. The chemiluminescent signals were either documented on film (Denville, Holliston, MA, USA) or by GE Amersham Imager model 600 (GE Healthcare, Pittsburgh, PA, USA). For protein bands documented on film, they were scanned by an Epson Perfection 2400 photo/film scanner (Long Beach, CA, USA) with at least 1200 d.p.i. resolution and then analyzed using Image $\mathrm{J}(\mathrm{NIH}$, Bethesda, MD, USA). For protein bands documented by the GE imager, an ImageQuant TL software pack (v8.1) (GE Healthcare, Pittsburgh, PA, USA) and its 1D gel analysis module was used. In addition, fluorophore-conjugated secondary antibodies (Li-COR, Lincoln, NE, USA), including anti-mouse and anti-rat, were used with a LICOR Odyssey imager to visualize protein bands through fluorescence.

Production of custom antibody against RDD peptide (antiRDD). Rabbit polyclonal anti-RDD was ordered from GenScript (Piscataway, NJ, USA) by custom antibody production protocol optimized for recognition of PTMs. In brief, a custom synthesized peptide with the sequence of RDDIAALVVDC (Genscript) was conjugated through the C-terminal cysteine to a carrier protein, keyhole limpet hemocyanin, to increase the presentation of the $\mathrm{N}$ terminus of the peptide. The conjugated protein was used as the immunogen for repeated immunization in rabbits. The collected antisera was cross-absorbed by another synthetic peptide with the sequence of DDIAALVVDC (Genscript). The resulted antisera (anti-RDD) was confirmed by immunblots to have minimal cross-reactivity to the peptide DDIAALVVDC, or the N-terminally acetylated peptide Ac-DDIAALVVDC (a gift from Dr. Anna Kashina in the University of Pennsylvania).

Microscopy. Optical and fluorescent imaging of cells were performed on a Zeiss Observer (Jena, Germany) equipped with a series of objectives and Zen Pro software (Carl Zeiss Microscopy, Jena, Germany).

Flow cytometry (FACS). FACS analysis or sorting was performed on a BD Canto-II flow cytometer (BD Biosciences, San Jose, CA, USA) at the core facility of the Sylvester Comprehensive Cancer Center at the University of Miami.

shRNA-mediated knockdown of Ate1. p.LKO lentiviral vectors containing shRNA targeting GFP, mouse Ate1 and human Ate1 were obtained from the Mission shRNA catalog (Sigma; clone NM_007041.1-520s1c1 for human; clone NM_013799.2-1507s1c1 for mouse). These vectors were packaged using VSV.g and $\Delta 8.2$ lentivirus packaging vectors by co-transfecting HEK293T cells with the aid of transfection kit Lipofectamin 2000 (Life Technologies). The viral supernatant was collected at 24, 48 and $72 \mathrm{~h}$, and filtered through a $0.45 \mu \mathrm{M}$ Syringe filter (Olympus, Genesee Scientific, San Diego, CA, USA, \#25-246). The supernatant was then used to transduce MEF and HFF cells, aided by $10 \mu \mathrm{g} / \mathrm{ml}$ polybrene. After transduction, $5 \mu \mathrm{g} / \mathrm{ml}$ puromycin was used to select transduced fibroblast cells for 5-10 days. Once the selected line was stabilized, a western blot was run on a cell lysate to examine changes in Ate1 levels.

Preparation of stably transfected mammalian cells. Retroviral vector pQC-XIG, and the corresponding vectors for viral core enzymes and surface packaging, GAG-Pol and VSV.g, was obtained from Addgene. Another retroviral vector pBabe-puro containing eGFP or mAte1.1-GFP is gift from Dr. Anna Kashina (UPenn). For virus production, these vectors were co-transfected into HEK293T cells with transfection kit Lipofectamin 2000 (Life Technologies). The viral supernatant was collected at 24, 48 and $72 \mathrm{~h}$, and filtered through a $0.45 \mu \mathrm{M}$ Syringe filter (Olympus, \#25-246). The supernatant was then used to transduce MEF, aided by $10 \mu \mathrm{g} / \mathrm{ml}$ polybrene. The stably transfected cells were then enriched by fluorescent sorting by the GFP signal.

Stress tests and radiation treatment of mammalian cells. To evaluate the survival of MEFs and HFFs to stress and radiation, $10^{4}$ cells were plated in each well of a 94-well black-wall plate, with four replicates per dose. The cells were allowed to attach for 4-6 h. For the $\mathrm{H}_{2} \mathrm{O}_{2}$ treatment, $\mathrm{H}_{2} \mathrm{O}_{2}$ (Sigma, Cat\# 323381) was added to the cells for $12 \mathrm{~h}$ or indicated durations. The cells were then washed with
PBS, and cell viability was assessed via Calcein AM staining. For the UV irradiation assay, the culture medium was removed immediately before the irradiation and immediately added back afterward. The cells were then allowed to recover for $24 \mathrm{~h}$ at $37^{\circ} \mathrm{C}$, in a $\mathrm{CO}_{2}$ incubator before examination or the next treatment. The cell viability was either assessed via staining of live-cell dye Calcein AM or by direct counting in an automated cell counter TC-20 (Bio-Rad) with the aid of cell viability dye Trypan blue.

Stress treatments of yeast cells. Plate-based yeast stress survival (serial dilution) for $\mathrm{NaCl}, \mathrm{CdCl}_{2}$ and high temperature was performed on YPD plates. $\mathrm{H}_{2} \mathrm{O}_{2}$ treatment was carried out by overlaying $\mathrm{H}_{2} \mathrm{O}_{2}$ on SD plates.

\section{Evaluation of yeast cell viability by colony-forming assay}

$\mathrm{H}_{2} \mathrm{O}_{2}$ treatment and cell survival: Cells in log phase growth were collected and suspended $\left(0.2 \mathrm{OD}_{600}\right)$ in $\mathrm{SD}$ medium containing various concentration of $\mathrm{H}_{2} \mathrm{O}_{2}$. The treatments were carried out for $3 \mathrm{~h}$ at $30^{\circ} \mathrm{C}$ with mechanical shaking. Cells were pelleted and washed with water and equal numbers of cells were plated on SD medium plates (containing no $\mathrm{H}_{2} \mathrm{O}_{2}$ ). Plates were kept at $30^{\circ} \mathrm{C}$ and colonies were counted after 5 days. CFUs with $0 \mathrm{mM} \mathrm{H}_{2} \mathrm{O}_{2}$ was set as $100 \%$ for normalization separately for each type of yeast.

$\mathrm{CdCl}_{2}$ treatment and cell survival: Cells in log phase growth were collected and suspended $\left(0.2 \mathrm{OD}_{600}\right)$ in $\mathrm{SD}$ medium containing $\mathrm{CdCl}_{2}$ as indicated in the main text, cultured at $30^{\circ} \mathrm{C}$ with mechanical shaking. At given time points, samples of yeast were withdrawn for measurement of $\mathrm{OD}$ and/or colony-forming assay. For colonyforming assay, cells were pelleted and washed with water and equal numbers of cells were plated on SD medium plates (containing no $\mathrm{CdCl}_{2}$ ). Plates were kept at $30^{\circ} \mathrm{C}$ and colonies were counted after 5 days. CFUs of yeast before the application of $\mathrm{CdCl}_{2}$ (time 0 ) was set as 100\% for normalization separately for each type of yeast.

Ate1 overexpression and cell survival: ate1 $\Delta$ cells harboring control plasmid or pYES2-pGAL1: ATE1 constructs with the URA3 selection gene marker were initially grown in glucose medium to reach log phase. Cells were then collected, washed and transferred to $2 \%$ raffinose medium and grown for $20 \mathrm{~h}$ at $30^{\circ} \mathrm{C}$ with mechanical shaking to deplete all glucose (which suppresses the pGAL1 promoter). Cells were then collected, washed and transferred to galactose medium, and grown for various times at $30{ }^{\circ} \mathrm{C}$ with mechanical shaking for the induction of protein expression driven by the pGAL1 promoter. Equal numbers of cells harboring control plasmid or pYES2-pGAL1: ATE1 were plated on Ura-minus, glucose-containing SD medium plates to terminate the galactose induction and measure colony formation abilities of yeast carrying the plasmid. Plates were kept at $30^{\circ} \mathrm{C}$ and colonies were counted after 5 days.

In vitro arginylation assay. For in vitro arginylation with yeast extract, exponential growth-phase cells of WT BY4741strain yeast (either transfected or not) were used. Equal number of cells treated and not treated with test conditions were lysed in arginylation reaction buffer (50 mM HEPES, pH 7.5, $25 \mathrm{mM} \mathrm{KCl,} 15 \mathrm{mM}$ $\mathrm{MgCl}_{2}, 2.5 \mathrm{mM}$ ATP, arginine $0.2 \mathrm{mM}$, PMSF $0.1 \mathrm{M}$ and yeast protease inhibitor cocktail (final dilution $100 \mathrm{x}$; Sigma-Aldrich). ${ }^{37}$ The ate $1 \Delta$ yeast expressing DD$\beta 15-G F P$ were also lysed in arginylation buffer. The WT cell lysate was mixed with equal volume of lysate of ate $1 \Delta$ cells expressing the DD- $\beta 15$-GFP to start the arginylation reaction. The reaction was carried out for $10 \mathrm{~min}$ at $37^{\circ} \mathrm{C}$. The reaction was stopped by the addition of $1 / 3$ volume of $4 \times$ SDS sample buffer and boiling.

For in vitro arginylation with extract of mammalian cells (transfected or not), exponential growth-phase cells of MEFs were collected from culture plate, washed by dPBS and then weighed on scale. The cell pellets were lysed with $2 x$ volume of a modified reaction buffer (50 mM TRIS/HCl, $32 \mathrm{mM} \mathrm{Na}_{3} \mathrm{PO}_{4}, \mathrm{pH} 7.4,5 \mathrm{mM} \mathrm{MgCl}$, $1 \mathrm{mM}$ EDTA, $2.5 \mathrm{mM}$ ATP, $0.2 \mathrm{mM}$ arginine and $0.2 \% \mathrm{NP}-40$ ). As an arginylation reporter, recombinant protein DD- $\beta 15$-GFP was expressed in ate $1 \Delta$ yeast and then purified by GFP-TRAPS nanobody conjugated to magnetic beads (Bulldog Bio, Portsmouth, NH, USA) and was shown to be $>95 \%$ pure by Coomassie-blue staining in SDS-PAGE. This protein was added to the cell lysate as the substrate for arginylation. The reaction was allowed to proceed at $\mathrm{RT}$ for $45 \mathrm{~min}$, and terminated by addition of equal volume of $4 \times$ SDS sample buffer and incubation in boiling-water bath for $10 \mathrm{~min}$.

Evaluation of mammalian cell viability and counting of viable cells by Calcein AM staining. Calcein AM (Life Technologies, Cat C1430) was used at a working concentration of $1 \mu \mathrm{g} / \mathrm{ml}$ in PBS to assess cell viability. Cells were incubated with Calcein AM for $30 \mathrm{~min}$, and fluorescence was measured on a Fluorostar Omega plate reader (BMG Labtech Inc., Cary, NC, USA). 
Reporter plasmid for testing emerging rate of mutant colonies among cells subjected to UV treatment:

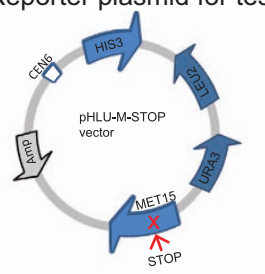

MET15 gene orginal sequence:

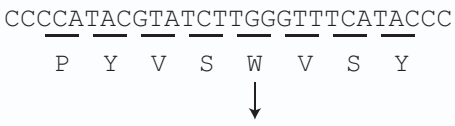

nucleotide

MET15-STOP sequence:

b

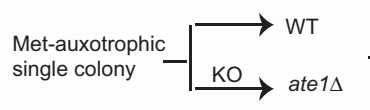

transform M-STOP

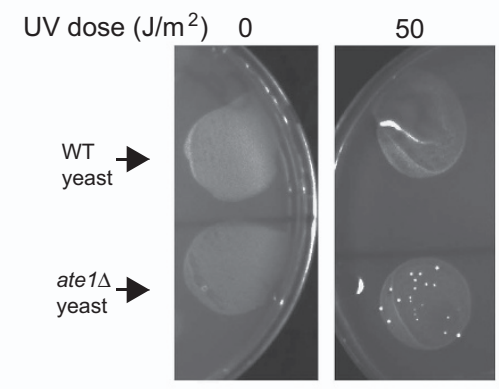

Met-minus plate

C

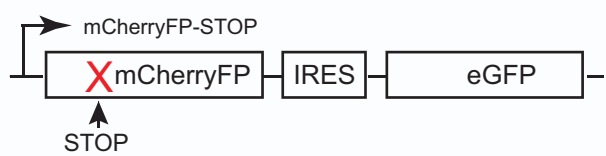

WT or Ate1-KO MEF stably tranfected with reporter $\rightarrow$ UV irradiation

d

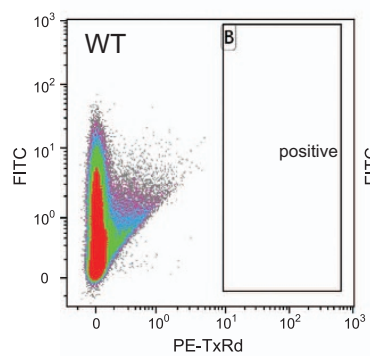

e

Ratio of red fluorescent cells (per million)

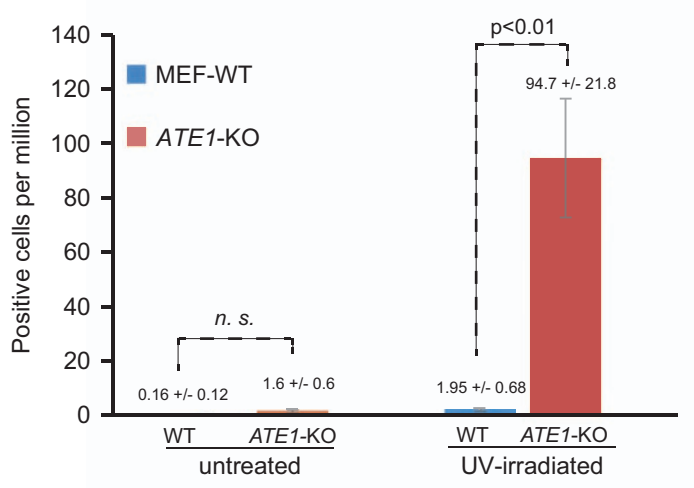

Direct counting of cell numbers. For cell number counting for yeast or mammalian cells, a TC-20 automated cell counter (Bio-Rad) was used. A viability dye Trypan blue, which only stains dead cells, was also used to facilitate the counting of mammalian cells to differentiate live and dead cells. plate equal

numbers of $\rightarrow$ UV exposure $\rightarrow$ measure Met-prototrophic cells on Metplate

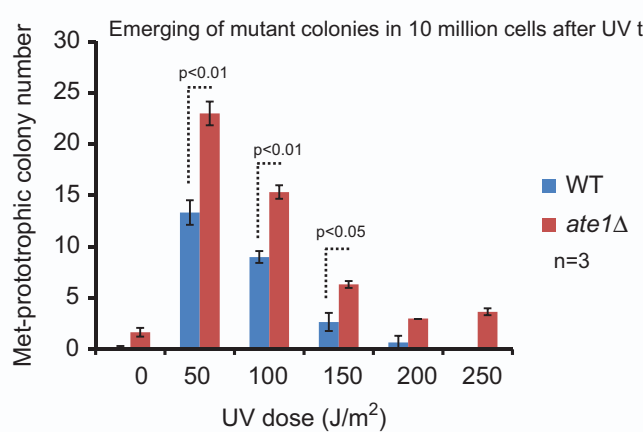

original mCherryFP: $\quad 286 \frac{\mathrm{TTC}}{\mathrm{F}} \frac{\mathrm{AAG}}{\mathrm{K}} \frac{\mathrm{TGG}}{\mathrm{W}} \frac{\mathrm{GAG}}{\mathrm{E}} \frac{\mathrm{CGC}}{\mathrm{R}} 300 \frac{\text { nucleotide }}{\text { amino acid }}$

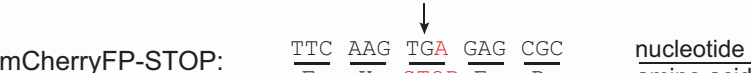
$\overline{\mathrm{F}} \overline{\mathrm{K}} \overline{\mathrm{STOP}} \overline{\mathrm{F}} \overline{\mathrm{R}} \quad \overline{\text { amino acid }}$

$\rightarrow$ Recover $\rightarrow$ FACS sorting

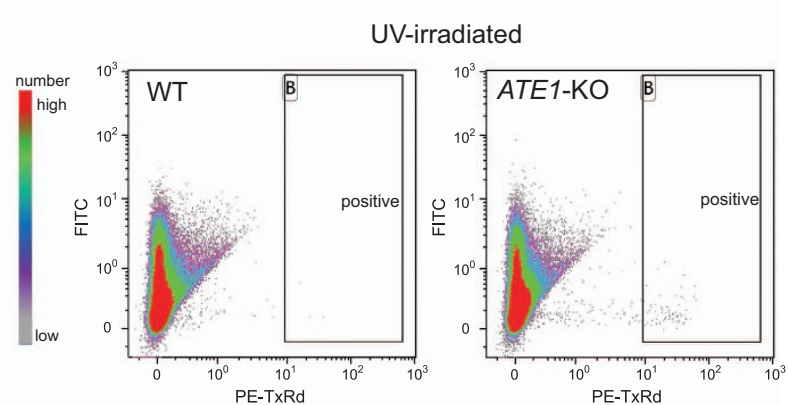

f Untreated ATE1-KO cells Irradiated and sorted ATE1-KO cells
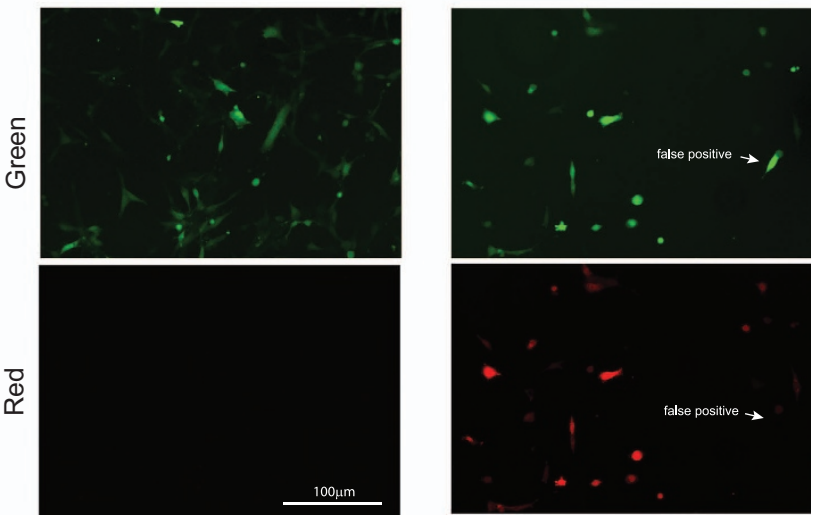

Evaluation of mammalian cell proliferation activity by EdU. Actively growing WT and ATE1-KO MEF cells were seeded at 10\% density and cultured for up to $18 \mathrm{~h}$ with final cell density of $<50 \%$ confluence. Click-iT EdU Alexa Fluor-594 Imaging kit (ThermoFisher Scientific, catalog number C10339) was used. 
Figure 8 Knockout of ATE1 increases mutagenesis upon UV irradiation. (a) Scheme showing the construction of a mutagenesis reporter plasmid. On the left is the vector map of the pHLUM-stop plasmid, which contains three auxotrophic marker genes: HIS3, LEU2, URA3 and a mutated MET15 gene with a stop codon in the middle of its coding sequence. The scheme on the right shows a portion of the coding sequences and corresponding amino acids in the original MET15 gene and the mutated Met15 stop gene, where a TGG codon, coding for tryptophan (W), is converted to a TGA stop codon. (b) The top panel shows a flow chart describing the procedure followed to create isogenic pairs of WT and ate1 $\Delta$ yeast and for testing emergence of Met-prototrophic mutant colonies on Met-minus plates starting with the same number of cells for UV irradiation. The bottom left panel has representative images showing the auxotrophic colonies emerged from 20 million yeast (in each spreading) without or with a low dose of UV exposure (50 $\left.\mathrm{J} / \mathrm{m}^{2}\right)$. The graph on the bottom right is the quantification of the experiment on the left for all tested doses of UV irradiations. Error bar represents S.E.M. ( $n=3$, except for the control nonirradiated groups where $n=6$ ). (c) Scheme showing the construction of a mammalian mutagenesis reporter, mCherryFP-STOP-IRES-GFP, which was modified from the pQCXIG retroviral vector suitable for stable transfection. A STOP codon is inserted in the N-terminal region of the mCherryFP-coding region so that this protein cannot be expressed as full-length, unless an acquired mutation reverts it to a sense codon (revertant). The scheme on the right shows a portion of the coding sequences and corresponding amino acids in the original mCherryFP gene and the mutated mCherryFP-STOP gene, where a TGG codon, coding for tryptophan (W), is converted to a TGA stop codon. (d) Representative FACS charts showing the distribution of cell populations by their green and red fluorescence, for WTor ATE1-KO MEF, in untreated condition or treated with lowdose UV irradiations that are not expected to lead to significant cell death (two pulses of $20 \mathrm{~J} / \mathrm{m}^{2}$ irradiations over $48 \mathrm{~h}$, followed by $24 \mathrm{~h}$ recovery). The windows marked 'B' were the gate setting used to quantify and sort red fluorescence-positive cells. (e) Quantification of mutated cells showing a red fluorescent signal in FACS in untreated or UV-irradiated cells from four independent repeats $(n=4)$. In untreated condition, both WTand ATE1-KO cells have negligible numbers of revertants with no significant (NS, $P>0.05$ ) difference. After UV irradiations, although the WT cells have a moderate increase of revertants ( 10 times), the increase in ATE1-KO is much larger ( 100 times), resulting in a significant difference between the WT and KO cells. Error bar represents S.E.M. As mentioned before, the $P$-value was calculated by Student's $t$-test. (f) Representative fluorescent images of ATE1-KO MEFs stably expressing the reporter genes, either untreated or treated with UV irradiation and enriched for red fluorescent cells by FACS for culturing of up to 1 week. In untreated cells, no red fluorescence presented in any examined cells. In UV-irradiated and sorted cells, the vast majority of the examined cells have prominent red fluorescence, in addition to the green fluorescence from the internal expression marker GFP on the reporter construct, indicating that they are true revertants. The white arrow in the image points to a false-positive cell, which only has green fluorescence and not red fluorescence. Overall, $<5 \%$ of false positive was found in the examined cells

The cells were pulse labeled with EdU for $45 \mathrm{~min}$ at $37^{\circ} \mathrm{C}$ in $5 \% \mathrm{CO}_{2}$ incubator, and then then washed with dPBS and fixed with $3.7 \%$ formaldehyde. The cells were then permeabilized with $0.5 \%$ Triton X-100 and incubated with the fluorescent labeling reaction cocktail supplied in the Click-iT EdU Imaging kit. Hoechst 33342, also supplied with the kit, was used for DNA staining for identification of cell nuclei as well as their morphologies. Only viable cells with normal nuclear morphologies and with no sign of apoptotic bodies were included in the final quantifications.

TUNEL assay for apoptosis. Exponential growth-phase cells (WT and Ate1 $\Delta$ ) were collected and suspended $\left(0.2 \mathrm{OD}_{600}\right)$ in $\mathrm{SD}$ medium containing $0,0.1$, 0.5 or $2 \mathrm{mM} \mathrm{H}_{2} \mathrm{O}_{2}$. The treatments were carried out for $3 \mathrm{~h}$ at $30^{\circ} \mathrm{C}$ with mechanical shaking. DNA strand breaks were examined by TUNEL with the In Situ Cell Death Detection kit, fluorescein (Roche Molecular Biochemicals, Branchburg, NJ, USA, \# 11684795910), as described earlier. Yeast cells were fixed with $3.7 \%$ (vol/vol) formaldehyde for $30 \mathrm{~min}$ at RT, washed three times with PBS and cell walls were digested with Lyticase at $2 \mathrm{U}$ per OD cells in $250 \mu \mathrm{l}$ cell suspension (Sigma-Aldrich, St. Louis, MO, USA, \# L2524-10KU) at $37^{\circ} \mathrm{C}$ for $1 \mathrm{~h}$. Ten microliters of the cell suspension was applied to a microscope slide and allowed to dry for $30 \mathrm{~min}$ at $37^{\circ}$ C. The slides were rinsed with PBS, incubated in permeabilization solution $(0.1 \%$ (vol/vol) Triton X-100 and 0.1\% (wt/wt) sodium citrate) for $2 \mathrm{~min}$ on ice, and rinsed twice with PBS. Slides were subsequently incubated with $10 \mu \mathrm{l}$ of TUNEL reaction mixture, containing terminal deoxynucleotidyl transferase and fluorescein isothiocyanate dUTP, for $60 \mathrm{~min}$ at $37^{\circ} \mathrm{C}$. Finally, the slides were rinsed three times with PBS, and a coverslip was mounted. Observations were carried out using Zeiss Observer equipped a series of objectives and the Zen Pro software.

Annexin-V staining assay for apoptosis. A total of $3 \times 10^{5} \mathrm{MEF}$ cells were seeded onto $60 \mathrm{mM}$ culture-treated dishes and allowed to adhere for $24 \mathrm{~h}$. Following indicated treatments, cells were trypsinized, washed with dPBS and pelleted. Annexin$\mathrm{V}$ and propidium iodide staining was accomplished using the Annexin-V FLUOS kit (Roche, 11858777001). Cells were resuspended in 100ul PBS with $1: 100$ Annexin-VFITC and 1 : 100 propidium iodide for $15 \mathrm{~min}$ at RT. Analysis of staining was performed on a BD Canto-II flow cytometer, using the FITC and propidium iodide channels.

UV irradiation. UV irradiations of yeast cells or mammalian cells was performed in a $254 \mathrm{~nm}$ UV-C irradiation oven (CL-1000S UV crosslinker from UVP, Upland, CA, USA) in a dark room.

Met-auxotrophy/prototrophy phenotype conversion assay of yeast. WT and Ate1 $\Delta$ yeast (BY4741 strain) were transformed with construct pHLUM-MET15-STOP (nonsense-trp318STOP). Yeast transformants were selected on SD medium containing methionine but not uracil. Transformants were grown in SD +methionine - uracil medium to log phase. Cultures were collected and washed three times with water and 20 million cells (in $100 \mu \mathrm{l}$ ) were spotted on SD plates (without methionine or Uracil). After any excessive liquid was absorbed by the plate, these cells were exposed to different doses of UV (ranging from 50 to $300 \mathrm{~J} / \mathrm{m}^{2}$ ), and then covered with aluminum foil immediately, all performed in the dark. Plates continued to be kept at RT in the dark to avoid triggering photoreactivation-related DNA repair mechanisms. Examination for the emergence of mutant colony began after three days of UV irradiations with continuous monitoring for up to 1 week.

UV-induced mutagenesis with dual-color reporter in mammalian cells. MEFs (WT or ATE1-KO) were stably transfected with the dual-color mutation reporter mCherryFP-STOP-IRES-GFP. Equal numbers of each type of cells were inoculated on $150 \mathrm{~mm}$-diameter cell culture dish for $4-6 \mathrm{~h}$ to allow attachment. The final cell density did not exceed $25 \%$ confluency. Immediately before UV irradiations, the culture medium was removed. After UV treatment, the original culture medium was added back to the dish and the cells were allowed to recover for $24 \mathrm{~h}$ in $\mathrm{CO}_{2}$ incubator at $37^{\circ} \mathrm{C}$ before another round of UV irradiation. After two rounds of UV irradiations, the cells were allowed to recover for $24 \mathrm{~h}$, before FACS analysis was performed on viable cells that remained attached to the dish.

Preparation of genomic DNA for Sanger sequencing. A total of 1-5 million of ATE1-KO cells carrying stably transfected mutation reporter mCherryFPSTOP-IRES-GFP, which was either untreated or UV-irradiated and sorted for red fluorescence, were used for genomic DNA extraction with Cyclo-Prep Genomic DNA isolation kit (Amresco, Solon, OH, USA) and proteinase $\mathrm{K}$ (New England Biolab, Ipswich, MA, USA). The region containing mCherryFP was amplified with high-fidelity polymerase Herculase (Agilent) in two rounds of nested PCR with four primer sets as described below:

To amplify the regions containing mCherryFP on the backbone of vector,

Forward primer targeting CMV promoter: AGAGCTCGTTTAGTGAACCGTC

Reverse primer targeting IRES: ACATATAGACAAACGCACACCG

PCR products with anticipated size from the first round were used as template for next round with these nested primers:

Xhol-CherryF: TTAACTCGAGATTGATCCGCATGGTG

Cherry-NotIR: TTAAGCGGCCGCCGGAATTTTACTTGTAC

The PCR products from the second round amplification with anticipated size were extracted by gel extraction kit (Qiagen, Valencia, CA, USA) and submitted for Sanger sequencing to Eurofins Genomics (Louisville, KY, USA) and Genewiz (South Plainfield, NJ, USA) for double confirmation of results.

\section{Conflict of Interest}

The authors declare no conflict of interest.

Acknowledgements. We thank Feng Gong (University of Miami) for providing access to the UV irradiation oven. We thank the Sylvester Comprehensive Cancer Center at the University of Miami for providing access to and subsidy for the flowcytometry core facilities. We thank Drs Sandra Lemmon, Kerry Burnstein, Feng Gong, Vlad Slepak, 
Murray Deutscher and Mary-Lou King (University of Miami), Anna Kashina (University of Pennsylvania), and Yan Gai (Saint Louis University) for critical reading of the manuscript. The cost of experiments and the salary of AK, MDB, DMP, WMM and FZ are supported in part by National Institutes of Health (NIH) R01 grants GM107333. AB is supported by NIH R01 grants: GM071775, GM105781 and GM112179. Patents pending for new techniques described in this manuscript for these authors: FZ, AK and MDB.

1. Kaji H, Novelli GD, Kaji A. A soluble amino acid-incorporating system from rat liver. Biochim Biophys Acta 1963; 76: 474-477.

2. Kwon YT, Kashina AS, Davydov IV, Hu RG, An JY, Seo JW et al. An essential role of $\mathrm{N}$-terminal arginylation in cardiovascular development. Science 2002; 297: 96-99.

3. Kurosaka S, Leu NA, Zhang F, Bunte R, Saha S, Wang J et al. Arginylation-dependent neural crest cell migration is essential for mouse development. PLOS Genet 2010; 6: e1000878.

4. Balzi E, Choder M, Chen WN, Varshavsky A, Goffeau A. Cloning and functional analysis of the arginyl-tRNA-protein transferase gene ATE1 of Saccharomyces cerevisiae. J Biol Chem 1990; 265: 7464-7471.

5. Kwon YT, Kashina AS, Varshavsky A. Alternative splicing results in differential expression, activity, and localization of the two forms of arginyl-tRNA-protein transferase, a component of the N-end rule pathway. Mol Cell Biol 1999; 19: 182-193.

6. Graciet E, Hu RG, Piatkov K, Rhee JH, Schwarz EM, Varshavsky A. Aminoacyl-transferases and the $\mathrm{N}$-end rule pathway of prokaryotic/eukaryotic specificity in a human pathogen. Proc Natl Acad Sci USA 2006; 103: 3078-3083.

7. Decca MB, Bosc C, Luche S, Brugière S, Job D, Rabilloud T et al. Protein arginylation in rat brain cytosol: a proteomic analysis. Neurochem Res 2006; 31: 401-409.

8. Wong CC, Xu T, Rai R, Bailey AO, Yates JR 3rd, Wolf YI et al. Global analysis of posttranslational protein arginylation. PLoS Biol 2007; 5: e258.

9. Wang YM, Ingoglia NA. N-terminal arginylation of sciatic nerve and brain proteins following injury. Neurochem Res 1997; 22: 1453-1459.

10. Bongiovanni G, Fissolo S, Barra HS, Hallak ME. Posttranslational arginylation of soluble rat brain proteins after whole body hyperthermia. J Neurosci Res 1999; 56: 85-92.

11. Xu NS, Chakraborty G, Hassankhani A, Ingoglia NA. N-terminal arginylation of proteins in explants of injured sciatic nerves and embryonic brains of rats. Neurochem Res 1993; 18: 1117-1123.

12. Chakraborty $\mathrm{G}$, Ingoglia NA. N-terminal arginylation and ubiquitin-mediated proteolysis in nerve regeneration. Brain Res Bull 1993; 30: 439-445.

13. Jack DL, Chakraborty G, Ingoglia NA. Ubiquitin is associated with aggregates of arginine modified proteins in injured nerves. Neuroreport 1992; 3: 47-50.

14. Luo D, Chakraborty G, Ingoglia NA. Post-translational modification of proteins by arginine and lysine following crush injury and during regeneration of rat sciatic nerves. Restor Neurol Neurosci 1990; 2: 53-61.

15. Shyne-Athwal S, Chakraborty G, Gage E, Ingoglia NA. Comparison of posttranslational protein modification by amino acid addition after crush injury to sciatic and optic nerves of rats. Exp Neurol 1988; 99: 281-295.

16. Chakraborty G, Leach $T$, Zanakis MF, Ingoglia NA. Posttranslational protein modification by amino acid addition in regenerating optic nerves of goldfish. J Neurochem 1986; 46: 726-732.

17. Shyne-Athwal S, Riccio RV, Chakraborty G, Ingoglia NA. Protein modification by amino acid addition is increased in crushed sciatic but not optic nerves. Science 1986; 231: 603-605.

18. Zanakis MF, Chakraborty G, Sturman JA, Ingoglia NA. Posttranslational protein modification by amino acid addition in intact and regenerating axons of the rat sciatic nerve. J Neurochem 1984; 43: 1286-1294.

19. Zhang N, Donnelly R, Ingoglia NA. Evidence that oxidized proteins are substrates for N-terminal arginylation. Neurochem Res 1998; 23: 1411-1420.

20. Klemperer NS, Pickart CM. Arsenite inhibits two steps in the ubiquitin-dependent proteolytic pathway. J Biol Chem 1989; 264: 19245-19252.

21. Ingoglia NA et al. What is the signal for the posttranslational arginylation of proteins? Neurochem Res 2000; 25: 51-58.

22. Bachmair A, Finley D, Varshavsky A. In vivo half-life of a protein is a function of its aminoterminal residue. Science 1986; 234: 179-186.

23. Piatkov Kl, Brower CS, Varshavsky A. The N-end rule pathway counteracts cell death by destroying proapoptotic protein fragments. Proc Natl Acad Sci USA 2012; 109: E1839-E1847.

24. Piatkov KI, Oh JH, Liu Y, Varshavsky A. Calpain-generated natural protein fragments as short-lived substrates of the N-end rule pathway. Proc Natl Acad Sci USA 2014; 111 E817-E826.

25. Brower CS, Piatkov KI, Varshavsky A. Neurodegeneration-associated protein fragments as short-lived substrates of the N-end rule pathway. Mol Cell 2013; 50: 161-171.

26. Xu Z, Payoe R, Fahlman RP. The C-terminal proteolytic fragment of the breast cancer susceptibility type 1 protein (BRCA1) is degraded by the $\mathrm{N}$-end rule pathway. J Biol Chem 2012; 287: 7495-7502.

27. Ditzel M, Wilson R, Tenev T, Zachariou A, Paul A, Deas E et al. Degradation of DIAP1 by the $\mathrm{N}$-end rule pathway is essential for regulating apoptosis. Nat Cell Biol 2003; 5: 467-473.

28. Varshavsky A. The N-end rule and regulation of apoptosis. Nat Cell Biol 2003; 5: 373-376.

29. Tasaki T, Sriram SM, Park KS, Kwon YT. The N-end rule pathway. Annu Rev Biochem 2012; $81: 261-289$
30. Eldeeb MA, Fahlman RP. The anti-apoptotic form of tyrosine kinase Lyn that is generated by proteolysis is degraded by the N-end rule pathway. Oncotarget 2014; 5: 2714-2722.

31. Karakozova M, Kozak M, Wong CC, Bailey AO, Yates JR 3rd, Mogilner A et al. Arginylation of beta-actin regulates actin cytoskeleton and cell motility. Science 2006; 313: 192-196.

32. Saha S, Mundia MM, Zhang F, Demers RW, Korobova F, Svitkina T et al. Arginylation regulates intracellular actin polymer level by modulating actin properties and binding of capping and severing proteins. Mol Biol Cell 2010; 21: 1350-1361.

33. Zhang F, Saha S, Kashina A. Arginylation-dependent regulation of a proteolytic product of talin is essential for cell-cell adhesion. J Cell Biol 2012; 197: 819-836.

34. Carpio MA, Decca MB, Lopez Sambrooks C, Durand ES, Montich GG, Hallak ME. Calreticulin-dimerization induced by post-translational arginylation is critical for stress granules scaffolding. Int J Biochem Cell Biol 2013; 45: 1223-1235.

35. Zhang F, Patel DM, Colavita K, Rodionova I, Buckley B, Scott DA et al. Arginylation regulates purine nucleotide biosynthesis by enhancing the activity of phosphoribosyl pyrophosphate synthase. Nat Commun 2015; 6: 7517.

36. Morano KA, Grant CM, Moye-Rowley WS. The response to heat shock and oxidative stress in Saccharomyces cerevisiae. Genetics 2012; 190: 1157-1195.

37. Wang J, Han X, Saha S, Xu T, Rai R, Zhang F et al. Arginyltransferase is an ATPindependent self-regulating enzyme that forms distinct functional complexes in vivo. Chem Biol 2011; 18: 121-130.

38. Li J, Pickart CM. Binding of phenylarsenoxide to Arg-tRNA protein transferase is independent of vicinal thiols. Biochemistry 1995; 34: 15829-15837.

39. Rai R, Kashina A. Identification of mammalian arginyltransferases that modify a specific subset of protein substrates. Proc Natl Acad Sci USA 2005; 102: 10123-10128.

40. Rai R, Zhang F, Colavita K, Leu NA, Kurosaka S, Kumar A et al. Arginyltransferase suppresses cell tumorigenic potential and inversely correlates with metastases in human cancers. Oncogene 2015; 35: 4058-4068.

41. Chandran UR, Ma C, Dhir R, Bisceglia M, Lyons-Weiler M, Liang W et al. Gene expression profiles of prostate cancer reveal involvement of multiple molecular pathways in the metastatic process. BMC Cancer 2007; 7: 64.

42. Varambally S, Yu J, Laxman B, Rhodes DR, Mehra R, Tomlins SA et al. Integrative genomic and proteomic analysis of prostate cancer reveals signatures of metastatic progression. Cancer Cell 2005; 8: 393-406.

43. Leu NA, Kurosaka S, Kashina A. Conditional Tek promoter-driven deletion of arginyltransferase in the germ line causes defects in gametogenesis and early embryonic lethality in mice. PLoS One 2009; 4: e7734.

44. Bongiovanni G, Fidelio GD, Barra HS, Hallak ME. The post-translational incorporation of arginine into a beta-amyloid peptide increases the probability of alpha-helix formation. Neuroreport 1995; 7: 326-328.

45. Rai R, Zhang F, Colavita K, Leu NA, Kurosaka S, Kumar A. Arginyltransferase suppresses cell tumorigenic potential and inversely correlates with metastases in human cancers. Oncogene 2015; 35: 4058-4068.

46. Masdehors $\mathrm{P}$, Glaisner S, Maciorowski Z, Magdelénat H, Delic J. Ubiquitin-dependent protein processing controls radiation-induced apoptosis through the $\mathrm{N}$-end rule pathway. Exp Cell Res 2000; 257: 48-57.

47. Brower CS, Rosen CE, Jones RH, Wadas BC, Piatkov KI, Varshavsky A. Liat1, an arginyltransferase-binding protein whose evolution among primates involved changes in the numbers of its 10-residue repeats. Proc Natl Acad Sci USA 2014; 111: E4936-E4945.

48. Deardorff MA, Bando M, Nakato R, Watrin E, Itoh T, Minamino M et al. HDAC8 mutations in Cornelia de Lange syndrome affect the cohesin acetylation cycle. Nature 2012; 489: 313-317.

49. Esashi F, Christ N, Gannon J, Liu Y, Hunt T, Jasin M et al. CDK-dependent phosphorylation of BRCA2 as a regulatory mechanism for recombinational repair. Nature 2005; 434: 598-604.

50. Kumar A, Tikoo S, Maity S, Sengupta S, Sengupta S, Kaur A et al. Mammalian proapoptotic factor $\mathrm{ChaC} 1$ and its homologues function as gamma-glutamyl cyclotransferases acting specifically on glutathione. EMBO Rep 2012; 13: 1095-1101.

51. Zhang F, Saha S, Shabalina SA, Kashina A. Differential arginylation of actin isoforms is regulated by coding sequence-dependent degradation. Science 2010; 329: 1534-1537.

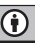

Cell Death and Disease is an open-access journal published by Nature Publishing Group. This work is licensed under a Creative Commons Attribution 4.0 International License. The images or other third party material in this article are included in the article's Creative Commons license, unless indicated otherwise in the credit line; if the material is not included under the Creative Commons license, users will need to obtain permission from the license holder to reproduce the material. To view a copy of this license, visit http://creativecommons.org/licenses/by/4.0/

\author{
(C) The Author(s) 2016
}

